A new open-source code for spherically symmetric stellar collapse to neutron stars and black holes

This article has been downloaded from IOPscience. Please scroll down to see the full text article.

2010 Class. Quantum Grav. 27114103

(http://iopscience.iop.org/0264-9381/27/11/114103)

View the table of contents for this issue, or go to the journal homepage for more

Download details:

IP Address: 131.215.220.165

The article was downloaded on 21/05/2010 at 23:04

Please note that terms and conditions apply. 


\title{
A new open-source code for spherically symmetric stellar collapse to neutron stars and black holes
}

\author{
Evan O'Connor ${ }^{1}$ and Christian D Ott ${ }^{1,2,3}$ \\ 1 TAPIR, Mail Code 350-17, California Institute of Technology, Pasadena, CA 91125, USA \\ 2 Niels Bohr International Academy, The Niels Bohr Institute, Copenhagen, Denmark \\ ${ }^{3}$ Center for Computation and Technology, Louisiana State University, Baton Rouge, LA, USA \\ E-mail: evanoc@tapir.caltech.edu and cott@tapir.caltech.edu
}

Received 8 December 2009, in final form 28 March 2010

Published 10 May 2010

Online at stacks.iop.org/CQG/27/114103

\begin{abstract}
We present the new open-source spherically symmetric general-relativistic (GR) hydrodynamics code GR1D. It is based on the Eulerian formulation of GR hydrodynamics (GRHD) put forth by Romero-Ibáñez-Gourgoulhon and employs radial-gauge, polar-slicing coordinates in which the $3+1$ equations simplify substantially. We discretize the GRHD equations with a finite-volume scheme, employing piecewise-parabolic reconstruction and an approximate Riemann solver. GR1D is intended for the simulation of stellar collapse to neutron stars and black holes and will also serve as a testbed for modeling technology to be incorporated in multi-D GR codes. Its GRHD part is coupled to various finite-temperature microphysical equations of state in tabulated form that we make available with GR1D. An approximate deleptonization scheme for the collapse phase and a neutrino-leakage/heating scheme for the postbounce epoch are included and described. We also derive the equations for effective rotation in 1D and implement them in GR1D. We present an array of standard test calculations and also show how simple analytic equations of state in combination with presupernova models from stellar evolutionary calculations can be used to study qualitative aspects of black hole formation in failing rotating core-collapse supernovae. In addition, we present a simulation with microphysical equations of state and neutrino leakage/heating of a failing corecollapse supernova and black hole formation in a presupernova model of a $40 M_{\odot}$ zero-age main-sequence star. We find good agreement on the time of black hole formation (within 20\%) and last stable protoneutron star mass (within 10\%) with predictions from simulations with full Boltzmann neutrino radiation hydrodynamics.
\end{abstract}

PACS numbers: $04.25 . \mathrm{D}-$, 04.40.Dg, 97.10.Kc, 97.60.Bw, 97.60.Jd, 97.60.Lf, 26.60.Kp

(Some figures in this article are in colour only in the electronic version) 


\section{Introduction}

Stellar core collapse is among the most energetic phenomena in the modern universe and liberates of the order of a few hundred $[\mathrm{B}]$ ethe $\left(1 \mathrm{~B}=10^{51} \mathrm{erg}\right)$ of gravitational energy as the core of a massive star (zero-age main-sequence (ZAMS) mass $8-10 M_{\odot} \lesssim M \lesssim 100 M_{\odot}$ ) is compressed from a radius of $\sim 1500 \mathrm{~km}$ and central density $\rho_{c} \sim 10^{10} \mathrm{~g} \mathrm{~cm}^{-3}$ to $\sim 15 \mathrm{~km}$ and $\rho_{c}$ in excess of nuclear density. Most $(\sim 99 \%)$ of this energy is ultimately radiated in neutrinos, but a small fraction $(\sim 1 \mathrm{~B})$ may be converted into kinetic and internal energy of an outgoing shock wave and may result in a core-collapse supernova explosion within the first seconds after collapse. The precise mode of conversion, the core-collapse supernova mechanism, is uncertain and has been the enigma of supernova theory for the past five decades (e.g. [1-8]). At the densities and velocities encountered in stellar collapse, the inclusion of general relativistic effects is not an optional model sophistication, but a necessity for quantitatively and qualitatively reliable results. Importantly, general relativity (GR) predicts that the protoneutron star (PNS) formed in the initial collapse will undergo a second gravitational instability and collapse to a black hole $(\mathrm{BH})$, if continued accretion pushes it over the maximum mass supported by the strong force and nucleon degeneracy. This may happen if the supernova mechanism fails and no explosion is launched or due to fallback accretion if an explosion occurs, but is too weak to unbind the entire stellar envelope [9]. In both cases, and provided sufficient angular momentum and its appropriate distribution in the progenitor star, the newly formed collapsar may become the central engine for a long-soft gamma-ray burst (GRB) [10, 11].

General relativistic computational models of stellar collapse have a long pedigree, starting with the spherically symmetric (1D) Lagrangian work of May and White in the mid-1960s [12], based on the comoving GR hydrodynamics formulation in orthogonal coordinates by Misner and Sharp [13] and using a finite-difference scheme with an artificial viscosity [14] approach to handle shocks. Much subsequent 1D GR work [15-20] was based on this or similar approaches, including full radiation-hydrodynamics stellar collapse and core-collapse supernova simulations with finite-temperature microphysical equations of state (EOS) [21-25]. Eulerian formulations, more suited for extension to multi-D simulations, were introduced later and used maximal slicing [26-29], or radial-gauge, polar-slicing (RGPS) [30]. These schemes, with the exception of [30], which employed pseudospectral methods, still used artificial viscosity approaches to shock treatment. More accurate, high-resolution shockcapturing (HRSC) approaches to GR stellar collapse based on higher order Godunov schemes and Riemann solvers were introduced by Marti et al [31] and Yamada [32] in the Lagrangian context, by Marti et al [33] in the fixed-background Eulerian case and by Romero et al [34] and Noble [35] in the RGPS Eulerian frame. Yamada's approach was later extended to include microphysical EOS and radiation transport [36, 37]. Gourgoulhon and Haensel [38] included an approximate neutrino transport treatment in their code. Preliminary results of Romero's code with a microphysical EOS and a neutrino leakage scheme were published in [39, 40].

State-of-the-art simulations of stellar collapse and of the postbounce supernova evolution strongly suggest that multi-D dynamics is crucial for the core-collapse supernova mechanism to succeed in massive stars (e.g. [2, 4, 41-44]). The present multi-D core-collapse supernova codes are either Newtonian [44-46] or employ Newtonian dynamics with relativistic corrections to the gravitational potential [3, 4, 7]. Multi-D simulations in conformally flat [47] or full GR traditionally relied on simple analytic EOS and polytropic initial models and neglected crucial neutrino effects (see, e.g., [48-51]). Only recently have the first axisymmetric (2D) $[52,53]$ and 3D [54, 55] GR core-collapse simulations become available that employ microphysical EOS and an approximate treatment of deleptonization in the collapse phase, 
but postbounce neutrino transport, cooling and heating are still not taken into account in these models. However, very recently, Müller [56] has succeeded in implementing the complex and computationally intensive radiation-transport scheme of [57] in the 2D conformally flat GR framework of [48, 49] and first results are forthcoming [58].

In this paper, we lay the foundations for a new and open approach to the stellar collapse and core-collapse supernova problem in GR. We discuss the formulation and implementation of the code GR1D, a new, spherically symmetric Eulerian GR code for stellar collapse to neutron stars and black holes with approximate pre- and postbounce neutrino treatment. We release GR1D and all its microphysics and input physics as an open source to be downloaded from http://www.stellarcollapse.org. It is meant to complement open-source 3D GR codes such as Whisky [59] that do not come with microphysics and neutrino approximations. At the same time, we intend GR1D to serve as an efficient 1D GR testbed for new modeling technology to be eventually incorporated in multi-D codes. In addition, GR1D and its microphysics components can readily be adapted for use in the computational modeling of problems involving some or much of the same physics as in the stellar collapse problem, e.g. the postmerger phase of double neutron star or black hole-neutron star coalescence.

We base GR1D on the conceptually simple and computationally efficient RGPS formalism of [30]. GR1D, like the code of [34], employs a Eulerian formulation of GR hydrodynamics with HRSC and works on non-equidistant grids. For the first time in the 1D GR context, we derive and implement in GR1D an extension of the 1D GR hydrodynamics equations to include rotation in an effective fashion. For completeness and comparison of Newtonian and GR dynamics, GR1D also implements 1D Newtonian hydrodynamics. GR1D operates with analytic EOS as well as with tabulated microphysical EOS through a general EOS interface. We discuss and provide EOS tables for the EOS of Lattimer-Swesty [60] and the one of Shen et al [61, 62]. Furthermore, we discuss and include in GR1D the deleptonization treatment of [63] for the collapse phase and a postbounce 3-flavor neutrino treatment based on the leakage schemes of $[64,65]$ as well as an approximate way of including neutrino heating.

Due to these approximations in the neutrino treatment, GR1D in its present form cannot be used for accurate simulations addressing the core-collapse supernova mechanism or neutrinoinduced nucleosynthesis. However, we find that with the present treatment, GR1D reproduces very well qualitatively the salient features of the postbounce evolution of core-collapse supernovae as predicted by full $1 \mathrm{D}$ radiation-hydrodynamics simulations. Moreover, we find that GR1D may be used to make quantitatively reliable predictions on the time of black hole formation in failing core-collapse supernovae and on the maximum mass of the PNS.

This paper is structured as follows. In section 2, we discuss our 1D GR hydrodynamics and curvature equations and their implementation in GR1D. Section 3 introduces the EOS provided with GR1D and in section 4 we detail our prebounce deleptonization and postbounce leakage and neutrino heating schemes. A number of code tests and example simulations are presented in section 5 and section 6. We wrap up and conclude in section 7.

We assume spacelike signature $(-,+,+,+)$ and, unless mentioned otherwise, use units of $G=c=M_{\odot}=1$, but use cgs units for the microphysics and neutrino leakage/heating quantities.

\section{1D GR hydrodynamics and curvature equations}

\subsection{Curvature equations in $1 D$ RGPS}

We follow [30, 34] which formulate the $3+1$ GR curvature and hydrodynamics equations in RGPS coordinates. In these coordinates and in spherical symmetry, the shift vector vanishes 
and the metric is diagonal and closely resembles the Schwarzschild metric. The invariant line element is

$$
\begin{aligned}
\mathrm{d} s^{2} & =g_{\mu \nu} \mathrm{d} x^{\mu} \mathrm{d} x^{\nu}, \\
& =-\alpha(r, t)^{2} \mathrm{~d} t^{2}+X(r, t)^{2} \mathrm{~d} r^{2}+r^{2} \mathrm{~d} \Omega^{2},
\end{aligned}
$$

where $\alpha$ and $X$ can be written more conveniently as functions of a metric potential, $\Phi(r, t)$, and the enclosed gravitational mass, $M_{\text {grav }}(r, t)=m(r, t)$,

$$
\alpha(r, t)=\exp [\Phi(r, t)], \quad X(r, t)=\left(1-\frac{2 m(r, t)}{r}\right)^{-1 / 2} .
$$

We assume ideal hydrodynamics for which the fluid stress-energy tensor and the matter current density are

$$
T^{\mu \nu}=\rho h u^{\mu} u^{\nu}+P g^{\mu \nu} \quad \text { and } \quad J^{\mu}=\rho u^{\mu},
$$

where $\rho$ is the baryonic density, $P$ is the fluid pressure and $h$ is the specific enthalpy equal to $1+\epsilon+P / \rho$ with $\epsilon$ being the specific internal energy. $u^{\mu}$ is the four-velocity and, in 1D without rotation, is equal to $\left[W / \alpha, W v^{r}, 0,0\right] . W=\left(1-v^{2}\right)^{-1 / 2}$ is the Lorentz factor and $v=X v^{r}$. The equation for the gravitational mass needed for determining the metric coefficient $X(r, t)$ of (2) is derived from the Hamiltonian constraint equation and reads

$$
m(r, t)=4 \pi \int_{0}^{r}\left(\rho h W^{2}-P+\tau_{m}^{\nu}\right) r^{\prime 2} \mathrm{~d} r^{\prime} .
$$

Here, $\tau_{m}^{\nu}$ is the contribution to the gravitational mass from the energy and pressure of trapped neutrinos (see section 4.3). The expression for the metric potential $\Phi(r, t)$ is determined via the momentum constraints, taking into account the polar slicing condition that imposes $\operatorname{tr} K=K_{r}{ }^{r}$, where $K_{i j}$ is the extrinsic curvature tensor (see [30, 35] for details). It reads

$$
\Phi(r, t)=\int_{0}^{r} X^{2}\left[\frac{m\left(r^{\prime}, t\right)}{r^{\prime 2}}+4 \pi r^{\prime}\left(\rho h W^{2} v^{2}+P+\tau_{\Phi}^{v}\right)\right] \mathrm{d} r^{\prime}+\Phi_{0},
$$

where analogous to (4) $\tau_{\Phi}^{v}$ accounts for the effect of trapped neutrinos. $\Phi_{0}$ is determined by matching the solution at the star's surface $\left(r=R_{\star}\right)$ to the Schwarzschild metric:

$$
\Phi\left(R_{\star}, t\right)=\ln \left[\alpha\left(R_{\star}, t\right)\right]=\frac{1}{2} \ln \left[1-\frac{2 m\left(R_{\star}, t\right)}{R_{\star}}\right] .
$$

We use standard second-order methods to perform the integrals in (4) and (5) and obtain values at cell centers as well as at cell interfaces.

\subsection{GR hydrodynamics in $1 D$ RGPS}

The evolution equations for the matter fields are derived from the local conservation laws for the stress-energy tensor, $\nabla_{\mu} T^{\mu \nu}=0$, and for the matter current density $\nabla_{\mu} J^{\mu}=0$. We write the GR hydrodynamics equations along the lines of the flux-conservative Valencia formulation (e.g. [66-68]) with modifications for spherically symmetric flows proposed by [34] and neutrino sources. Derivation details are presented in appendix A.

We write the set of evolution equations as

$$
\partial_{t} \vec{U}+\frac{1}{r^{2}} \partial_{r}\left[\frac{\alpha r^{2}}{X} \vec{F}\right]=\overrightarrow{\mathcal{S}}
$$

where $\vec{U}$ is the set of conserved variables, $\vec{F}$ is their flux vector and $\overrightarrow{\mathcal{S}}$ is the vector containing gravitational, geometric and neutrino-matter interaction sources and sinks. In $1 \mathrm{D}$ and without 
rotation, $\vec{U}=\left[D, D Y_{e}, S^{r}, \tau\right]$. The conserved variables are functions of the primitive variables $\rho, Y_{e}, \epsilon, v$, and $P$ and are given by

$$
\begin{aligned}
& D=\alpha X J^{t}=X \rho W, \\
& D Y_{e}=\alpha X Y_{e} J^{t}=X \rho W Y_{e}, \\
& S^{r}=\alpha X T^{t r}=\rho h W^{2} v, \\
& \tau=\alpha^{2} T^{t t}-D=\rho h W^{2}-P-D,
\end{aligned}
$$

where $Y_{e}$ is the electron fraction, the number of electrons per baryon, and the only compositional variable needed to describe matter in nuclear statistical equilibrium (NSE). Note that there is a misprint in the central part of equation 9 of [34] which is missing a factor of $X$ which we have corrected here. The flux $\vec{F}$ is given by $\vec{F}=\left[D v, D Y_{e} v, S^{r} v+P, S^{r}-D v\right]$ and the sources and sinks are given by

$$
\begin{aligned}
\overrightarrow{\mathcal{S}}=\left[0, R_{Y_{e}}^{v},(\right. & \left.S^{r} v-\tau-D\right) \alpha X\left(8 \pi r P+\frac{m}{r^{2}}\right)+\alpha P X \frac{m}{r^{2}} \\
+ & \left.\frac{2 \alpha P}{X r}+Q_{S^{r}}^{v, \mathrm{E}}+Q_{S^{r}}^{v, \mathrm{M}}, Q_{\tau}^{v, \mathrm{E}}+Q_{\tau}^{v, \mathrm{M}}\right] .
\end{aligned}
$$

The source and sink terms $R_{Y_{e}}^{\nu}, Q_{S^{r}}^{\nu, \mathrm{E}}, Q_{S^{r}}^{\nu, \mathrm{M}}, Q_{\tau}^{\nu, \mathrm{E}}$ and $Q_{\tau}^{\nu, \mathrm{M}}$ are associated with neutrinos and are discussed in section 4 and derived in appendix A.

We use a semi-discrete approach and first discretize (7) in space, then apply the method of lines (MoL, [69]) and perform the time integration of the conserved variables via standard second- or third-order Runge-Kutta integrators with a Courant factor of 0.5.

The spatial discretization follows the finite-volume approach (e.g. [34, 68]) and all variables are defined at cell centers $i$ and must be reconstructed (i.e. interpolated) at cell interfaces, where inter-cell fluxes are computed. This interpolation must be monotonic to ensure stability. We use the nominally third-order (in smooth parts of the flow) piecewise-parabolic method (PPM, [70]) to interpolate the primitive variables and then set up the conserved variables at the cell interfaces. We also implement piecewise-constant reconstruction as well as piecewise-linear (total-variation-diminishing (TVD)) reconstruction with Van Leer's limiter [71]. The latter we use exclusively in the innermost three to five zones to avoid oscillations near the origin.

Once the variables have been reconstructed at the cell interfaces, we evaluate the physical interface fluxes $\vec{F}_{i+1 / 2}$ with the HLLE Riemann solver [72]. The right-hand-side (RHS) flux update term for $\vec{U}_{i}$ then reads

$$
\mathrm{RHS}_{i}=-\frac{1}{r_{i}^{2} \Delta r_{i}}\left[\frac{\alpha_{i+1 / 2} r_{i+1 / 2}^{2}}{X_{i+1 / 2}} \vec{F}_{i+1 / 2}-\frac{\alpha_{i-1 / 2} r_{i-1 / 2}^{2}}{X_{i-1 / 2}} \vec{F}_{i-1 / 2}\right] .
$$

Gravitational, geometrical and neutrino-matter interaction sources/sinks are not taken into account in the flux computation and are coupled into the MoL integration.

After the update of the conserved variables $D, D Y_{e}, S^{r}$ and $\tau$, primitive variables $\rho, Y_{e}, v$, $\epsilon$ and $P\left(\rho, \epsilon, Y_{e}\right)$ must be extracted since they are needed for the next timestep. In the general case, the primitive variables (with the exception of $Y_{e}$ ) cannot be expressed algebraically in terms of the conserved variables (see, e.g., [67]). Hence, we employ an iterative approach and make an initial guess using $P_{\text {old }}$ from the previous timestep:

$$
v=\frac{S^{r}}{\tau+D+P_{\text {old }}} \quad \rho=\frac{D}{X W}, \quad \epsilon=\frac{\tau+D+P_{\text {old }}\left(1-W^{2}\right)}{\rho W^{2}}-1,
$$

where we note that $X$ can be calculated from the conserved variables as $\rho h W^{2}-P=\tau+D$. $W$ is calculated from the estimate of $v$. We then call the EOS to obtain a new pressure and 
iterate this process using a Newton-Raphson method until convergence (we typically stop the iteration at a fractional pressure difference of $10^{-10}$ between iteration steps).

\subsection{Extension to 1.5D: including rotation}

Lagrangian spherically symmetric stellar evolution codes have long included rotation and rotational effects in an approximate fashion (e.g. [73-75]). The way this is typically done is to make the assumption that the star has constant angular velocity on spherical shells. In order to compute the effective specific centrifugal force acting on a fluid parcel, we compute the angular average of $(\vec{\omega} \times \vec{r})^{2}$ on a spherical shell of radius $r$, which leads to $f_{\text {cent }}=2 / 3 \omega^{2} r$. In Newtonian Lagrangian calculations, specific angular momentum $j=\omega r^{2}$ is conserved by construction and the effective centrifugal force appears in the momentum equation. Relatively recently, such an approach has also been taken in the Newtonian 1D core-collapse calculations of [76, 77] in order to take into account the effect of rotation approximately. In the Eulerian frame and in GR the situation is more complicated. We must solve an equation for angular momentum conservation on top of taking into account a centrifugal force term in the momentum equation. We begin by defining an azimuthal Eulerian velocity $v^{\phi}(=\omega)$ and, in order to obtain a quantity of dimension velocity, we also define $v_{\varphi}=r v^{\phi}$ (note that $u^{\phi}=W v_{\varphi} / r$ ). With finite $v^{\phi}, T^{r \phi}$ is finite and $W$ becomes $W=\left(1-v^{2}-2 / 3 v_{\varphi}^{2}\right)^{-1 / 2}$ in our effective approach. We provide derivation details in appendix A. 2 and present here only the results. The modified stress-energy tensor leads to an additional equation for angular momentum conservation analogous to (7):

$$
\partial_{t}\left(S_{\phi}\right)+\frac{1}{r^{2}} \partial_{r}\left(\frac{\alpha r^{2}}{X} F_{\phi}\right)=\mathcal{S}_{\phi}
$$

where

$$
\begin{aligned}
S_{\phi} & =\rho h W^{2} v_{\varphi} r, \\
F_{\phi} & =\rho h W^{2} v_{\varphi} r v=S_{\phi} v, \\
\mathcal{S}_{\phi} & =\rho h W^{2} \alpha v v_{\varphi} X\left[4 \pi r^{2} P+\frac{m}{r}\right] .
\end{aligned}
$$

Also, an additional term, accounting for the centrifugal force,

$$
+\alpha \frac{2}{3}\left(\frac{\rho h W^{2} v_{\varphi}^{2}}{X r}\right)
$$

appears on the RHS of the equation for $S^{r}$. Finally, the change of the stress-energy tensor also has an effect on the metric potential $\Phi$ whose equation is now given by

$$
\partial_{r} \Phi=X^{2}\left[\frac{m}{r^{2}}+4 \pi r\left(\rho h W^{2}\left(v^{2}+\frac{2}{3} v_{\varphi}^{2}\right)+P+\tau_{\Phi}^{v}\right)\right] .
$$

We implement this 1.5D treatment of rotation in GR1D, but keep the metric diagonal. The 1.5D treatment should be rather accurate for slow rotation, and, as shown by [77], will still capture qualitatively the effect of centrifugal support due to rapid rotation. For completeness, we note that the total angular momentum of the system (see, e.g., [78]) is given by

$$
J=\int_{0}^{\infty} T_{\phi}^{t} \sqrt{-g} \mathrm{~d}^{3} x=\frac{8 \pi}{3} \int_{0}^{\infty} \rho h X W^{2} r v_{\varphi} r^{2} \mathrm{~d} r,
$$

where we include a factor of $2 / 3$ to account for the angular average. The rotation parameter $\beta$, defined as the ratio $T /\left|W_{\text {grav }}\right|$ of rotational kinetic to gravitational energy, is

$$
T /\left|W_{\text {grav }}\right|=\frac{T}{\left|M_{\text {grav }}-M_{\text {proper }}-T\right|},
$$


where

$$
T=\frac{1}{2} \int_{0}^{\infty} \omega T_{\phi}^{t} \sqrt{-g} \mathrm{~d}^{3} x=\frac{4 \pi}{3} \int_{0}^{\infty} \rho h X W^{2} v_{\varphi}^{2} r^{2} \mathrm{~d} r
$$

where again in the last step averaging over the angular variables produces a factor of $2 / 3$. $M_{\text {proper }}$ is given by

$$
M_{\text {proper }}=4 \pi \int_{0}^{\infty}(\rho+\rho \epsilon) X W r^{2} \mathrm{~d} r
$$

and $M_{\text {grav }}$ is specified by (4).

\section{Equations of state (EOS)}

An EOS is needed to close the system of GR hydrodynamics equations and provide the pressure as well as other thermodynamic quantities as a function of density, temperature (or specific internal energy) and composition. In GR1D, we include for test simulations the standard analytic polytropic (isentropic 'cold', $P=K \rho^{\Gamma}$ ) and the $\Gamma$-law EOS ('hot', $P=(\Gamma-1) \rho \epsilon)$. These are inappropriate for stellar collapse since they do not capture the stiffening of the EOS at nuclear density. An analytic EOS, able to capture this effect qualitatively and include nonisentropic effects, is the hybrid EOS [79] which we include in GR1D and discuss in section 3.1. For a more realistic description of the thermodynamics of nuclear matter, an EOS built from a microphysical finite-temperature model for nuclear matter is needed. This is also a prerequisite for any kind of neutrino treatment, since crucial compositional information as well as chemical potentials must be derived from a microphysical model. Such microphysical EOS are too complicated to be computed on the fly in a simulation and are used in tabulated form with interpolation. GR1D is able to handle such EOS and we provide tables at http://www.stellarcollapse.org/microphysics for the EOS of Lattimer and Swesty ([60], LS EOS) and for the one of H. Shen et al ([61, 62], HShen EOS). The details of these tables and the routines facilitating their use are discussed in sections 3.2 and 3.3.

\subsection{Hybrid EOS}

The hybrid EOS found widespread use in early multi-D simulations of rotating core collapse (e.g. $[48,80])$, but was shown by $[52,53]$ to lead in some cases to qualitatively incorrect results for the collapse dynamics and the resulting gravitational wave signal. We include it in GR1D because its analytic nature provides for very fast calculations, allowing us to readily test the GR hydrodynamics of GR1D.

The hybrid EOS splits the pressure into a polytropic (cold) and a thermal component:

$$
P=P_{\text {cold }}+P_{\text {thermal }} \text {. }
$$

The cold part is piecewise polytropic. It is composed of a polytropic EOS with $\Gamma=\Gamma_{1}$ for densities below nuclear $\left(\rho_{\text {nuc }}\right)$ and another polytropic EOS with $\Gamma=\Gamma_{2}$ for densities above $\rho_{\text {nuc }}$. The two are smoothly matched at $\rho_{\text {nuc }}$ which makes the polytropic constant $K_{2}$ of the high-density part a function of the two $\Gamma \mathrm{s}$, of $K_{1}$, and of the transition density $\rho_{\text {nuc }}$ (see, e.g., [79-81] for a description of the procedure and detailed expressions). The thermal part is modeled via a $\Gamma$-law with $\Gamma_{\text {th }}$. It becomes relevant only after core bounce when shocks are present, making the flow nonadiabatic. Its contribution is determined via the thermal specific internal energy which is the difference between the primitive variable $\epsilon$ and the cold specific internal energy, $\epsilon_{\text {th }}=\epsilon-\epsilon_{\text {cold }}$. 
For collapse simulations, we set $K_{1}=1.2435 \times 10^{15}\left(Y_{e}\right)^{4 / 3}$ [cgs] (the value appropriate for a relativistic degenerate gas of electrons, [80, 82]) with $Y_{e}=0.5$. We choose a value below, but close to $4 / 3$ for $\Gamma_{1}$ and typically set $\Gamma_{2}=2.5$ to mimic the stiff nuclear EOS above $\rho_{\text {nuc }}$ which we set to $2 \times 10^{14} \mathrm{~g} \mathrm{~cm}^{-3}$. $\Gamma_{\text {th }}$ we normally keep at 1.5 to model a mixture of relativistic $(\Gamma=4 / 3)$ and nonrelativistic $(\Gamma=5 / 3)$ thermal contributions. This leads to rapid shock propagation and explosion. When simulating $\mathrm{BH}$ formation with the hybrid EOS, we set $\Gamma_{\text {th }}$ to smaller values. This reduces the postshock thermal pressure and leads to shock stagnation.

\subsection{Lattimer-Swesty EOS}

The LS EOS [60] is derived from a finite-temperature compressible liquid-droplet model [83] with a Skyrme nuclear force, uses the single heavy nucleus approximation and assumes nuclear statistical equilibrium (NSE). NSE holds at $T \gtrsim 0.5 \mathrm{MeV}$ which in core collapse and supernova matter is typically the case at $\rho \gtrsim$ few $\times 10^{7} \mathrm{~g} \mathrm{~cm}^{-3}$.

The LS EOS routines are open source and available from the Stony Brook group ${ }^{4}$. We employ their baryonic parts to generate tables with nuclear incompressibilities $K_{0}$ of $180 \mathrm{MeV}$, $220 \mathrm{MeV}$ and $375 \mathrm{MeV}$ (the larger the $K_{0}$, the stiffer the nuclear EOS). Hereafter, we refer to these $K_{0}$-variants of the LS EOS as LS180, LS220 and LS375. The symmetry energy $S_{v}$ is set in all variants to $29.3 \mathrm{MeV}$. Electrons and photons are added using the routines provided by Timmes' $\operatorname{EOS}^{5}$ [84].

We compute the maximum cold neutron star masses for the three LS EOS variants by setting $T=0.1 \mathrm{MeV}$ and assuming neutrino-less $\beta$-equilibrium. The results are $1.83 M_{\odot}$ $\left(2.13 M_{\odot}\right), 2.04 M_{\odot}\left(2.41 M_{\odot}\right)$ and $2.72 M_{\odot}\left(3.35 M_{\odot}\right)$ for gravitational (baryonic) mass and for $K_{0}=180 \mathrm{MeV}, K_{0}=220 \mathrm{MeV}$, and $K_{0}=375 \mathrm{MeV}$, respectively. The coordinate radii of these maximum mass stars are $10.1 \mathrm{~km}, 10.6 \mathrm{~km}$ and $12.3 \mathrm{~km}$.

Our LS EOS tables have 18 evenly spaced points per decade in $\log _{10} \rho$ ranging from $10^{3}$ to $10^{16} \mathrm{~g} \mathrm{~cm}^{-3}, 30$ points per decade in $\log _{10} T$ ranging from $10^{-2}$ to $10^{2.4} \mathrm{MeV}$ and 50 points equally spaced in electron fraction from 0.035 to 0.53 . This table resolution is sufficiently good to allow the use of simple and fast tri-linear interpolation $\left(\right.$ in $\left.\log _{10}(\rho), \log _{10}(T), Y_{e}\right)$ in collapse simulations while maintaining good thermodynamic consistency. In tests of adiabatic collapse, the inner-core entropy is conserved to $\sim 1 \%$ from the onset of collapse to core bounce.

To generate the LS EOS tables, we employ the LS EOS at densities above $10^{8} \mathrm{~g} \mathrm{~cm}^{-3}$, but, due to unreliable convergence, use linear extrapolation of the Helmholtz free energy $F$ in $Y_{e}$ for $Y_{e}>0.5$ and in $T$ at $T<0.06 \mathrm{MeV}$. Note that the latter is far away from NSE, but is never reached by core-collapse trajectories at $\rho>10^{8} \mathrm{~g} \mathrm{~cm}^{-3}$. At densities below $10^{8} \mathrm{~g} \mathrm{~cm}^{-3}$, we use the Timmes EOS [84] and assume that the matter is an ideal gas composed of electrons, photons, neutrons, protons, alpha particles and heavy nuclei with the average $A$ and $Z$ given by the LS EOS at the transition.

Since the specific internal energies returned by the baryonic part of the Timmes EOS do not contain the nuclear binding energy, we shift the zero point of the Timmes EOS so that the returned specific internal energies are consistent with the LS EOS values at the transition point. For simplicity, we keep baryonic compositional variables fixed at the values obtained from the LS EOS at the transition density. These particular choices for the baryonic component have little effect at low densities where the thermodynamics are dominated by electrons at low to intermediate temperatures and by photons at high temperatures. However, for full corecollapse supernova simulations that intend to address also nuclear burning and nucleosynthesis

4 http://www.astro.sunysb.edu/dswesty/lseos.html

$5 \mathrm{http}: / /$ cococubed.asu.edu/code_pages/eos.shtml 
aspects, a more involved consistent NSE/non-NSE EOS treatment involving the advection of many chemical species and a treatment of their interactions with a nuclear reaction network is necessary. We will leave such a treatment to future work (but see, e.g., [57, 85] for discussions of such implementations).

When using finite-temperature microphysical NSE EOS such as the LS EOS in GR hydrodynamics codes, two additional caveats need to be taken into account: (1) the thermodynamic potential from which all dependent variables are derived is the Helmholtz free energy $F$. This makes the EOS a function of $\left\{\rho, T, Y_{e}\right\}$ while GR hydrodynamics codes such as GR1D operate on the primitive thermodynamic and compositional variables $\left\{\rho, \epsilon, Y_{e}\right\}$. Hence, in a typical EOS call it is first necessary to determine $T\left(\rho, \epsilon, Y_{e}\right)$ through a root-finding procedure, before the dependent variables can be obtained through tri-linear interpolation in $\left\{\rho, T, Y_{e}\right\}$. (2) In contrast to Newtonian hydrodynamics that involves only differences of the specific internal energy $\epsilon$, GR codes depend directly on $\epsilon$ through its contribution to the matter stress-energy tensor. Hence, it is important to find and use a physically correct energy zero point and ensure that there are no rest-mass contributions included in $\epsilon$.

\subsection{HShen EOS}

The HShen EOS $[61,62]$ is based on a relativistic mean-field model for nuclear interactions, assumes NSE and is extended with the Thomas-Fermi approximation to describe the homogeneous phase of matter as well as the inhomogeneous matter composition. $K_{0}$ of the HShen EOS is $281 \mathrm{MeV}$ and the symmetry energy $S_{v}$ has a value of $36.9 \mathrm{MeV}$. The authors of the HShen EOS provide the baryonic component ${ }^{6}$ in tabulated form only. The provided table is not uniformly spaced and has too low resolution to be used directly with fast tri-linear interpolation in simulations. Hence, we generate a finer uniformly spaced table that has 18 points per decade in $\log _{10} \rho$ from $10^{3}$ to $10^{15.36} \mathrm{~g} \mathrm{~cm}^{-3}, 41$ points per decade in $\log _{10} T$ from $10^{-2}$ to $10^{2.4} \mathrm{MeV}$ and 50 points in $Y_{e}$ covering the interval $0.015-0.56$. We interpolate all dependent variables from the original HShen table using the cubic Hermite interpolation function given in [86] modified to have monotonic interpolation behavior according to the prescription of [87]. The interpolation is performed first bicubic in $\rho, T$, and then cubic in $Y_{e}$. Alternatively to the just described, one could interpolate the Helmholtz free energy $F$ and re-derive dependent variables by taking derivatives of $F$ on the interpolated table (see, e.g., [86]). We decided against this approach, since it would require quintic interpolation and the knowledge of the second derivatives of $F$ at each point in the original table, some of which would have to be computed by taking second derivatives in the coarse original table. Also, compositional information cannot be obtained directly from $F$ and would have to be interpolated from the original table.

We perform the described interpolation at densities above $10^{7} \mathrm{~g} \mathrm{~cm}^{-3}$. For points with $T>100 \mathrm{MeV}$ and $T<0.1 \mathrm{MeV}$ we extrapolate most variables linearly, keeping only the compositions fixed. We add photons and electrons after interpolation using the routines of the Timmes EOS. At densities below $10^{7} \mathrm{~g} \mathrm{~cm}^{-3}$, we employ the Timmes EOS in the same fashion as described above for the LS EOS.

We compute the maximum cold neutron star masses for the HShen EOS in the same way as for the LS EOS and find $2.24 M_{\odot}$ and 2.61 $M_{\odot}$ for the gravitational and baryonic values, respectively. The coordinate radius of the corresponding star is $12.6 \mathrm{~km}$.

6 http://user.numazu-ct.ac.jp/ sumi/eos 


\section{Neutrino leakage and heating}

\subsection{Deleptonization and electron capture in the collapse phase}

Electron capture on free and bound protons leads to the emission of neutrinos that stream away from the core and carry away net lepton number at densities below $\sim 10^{12} \mathrm{~g} \mathrm{~cm}^{-3}$. Hence, one speaks of the deleptonization of the core. The change of the electron fraction $Y_{e}$ in the collapse phase due to deleptonization has important dynamical consequences. A reduction of $Y_{e}$ leads to a decrease of the mass of the homologously collapsing inner core whose kinetic energy is initially imparted on the supernova shock and which turns into the PNS core after bounce [1]. We take electron capture in collapse into account in GR1D with the approximate scheme of Liebendörfer [63] who observed that $Y_{e}$ of infalling mass elements depends primarily on the local matter density $\rho$ and can be parameterized with rather high precision on the basis of radiation-hydrodynamic calculations.

Operator-split, after a hydrodynamics update, we compute the change in $Y_{e}$ :

$$
\Delta Y_{e}=\min \left[0, \bar{Y}_{e}(\rho)-Y_{e}\right]
$$

which ensures for consistency that a change in $Y_{e}$ is either negative or 0 . We use for $\bar{Y}_{e}(\rho)$ the fitting formula given in [63] with parameters $\rho_{1}=3 \times 10^{7} \mathrm{~g} \mathrm{~cm}^{-3}, \rho_{2}=2 \times 10^{13} \mathrm{~g} \mathrm{~cm}^{-3}$, $Y_{1}=0.5, Y_{2}=0.278$ and $Y_{c}=0.035$ corresponding to the $15 M_{\odot}$ model of [88], evolved as model G15 by [2]. GR1D also contains an interpolation routine to use numerical $\bar{Y}_{e}(\rho)$ data.

Electron capture leads to a change in the entropy ( $s$, the specific entropy in units of $k_{\mathrm{B}}$ /baryon) that is carried away by neutrinos leaving the core at densities below an assumed trapping density $\rho_{\text {trap }}=2 \times 10^{12} \mathrm{~g} \mathrm{~cm}^{-3}$. The entropy change is given by

$$
\Delta s=-\Delta Y_{e} \frac{\mu_{p}-\mu_{n}+\mu_{e}-E_{v}}{k_{\mathrm{B}} T} .
$$

$E_{v}$ is the energy of the escaping neutrinos (set to $10 \mathrm{MeV}$ ). $\mu_{p}, \mu_{n}$ and $\mu_{e}$ are the proton, neutron and electron chemical potentials including rest mass, respectively. Following [63], we set $\Delta s=0$ if $\mu_{p}+\mu_{n}+\mu_{e}<E_{v}$ and above $\rho_{\text {trap }}$. After updating the entropy, we use the EOS to update the specific internal energy $\epsilon$ for consistency with the new $Y_{e}$ and $s$.

We employ the outlined deleptonization scheme until core bounce (defined as the time when the peak entropy of the inner core surpasses $3 k_{\mathrm{B}}$ /baryon) and until $5 \mathrm{~ms}$ after bounce for yet unshocked regions of the outer core that will settle in the high-density outer PNS and only in this way assume realistic postbounce Ye.

\subsection{Postbounce deleptonization and neutrino heating/cooling}

At core bounce a strong hydrodynamic shock wave is generated that travels outward into the outer core, heating and dissociating infalling heavy nuclei into nucleons. Electron capture occurs rapidly on free protons and a sea of electron neutrinos $\left(v_{e}\right)$ builds up and is released in the $v_{e}$ burst when the shock breaks through the neutrinosphere ${ }^{7}$, deleptonizing the postshock region and leaving behind a 'trough' in the $Y_{e}$ profile (e.g. [41]). The softening of the EOS due to dissociation of nuclei and postshock energy loss to escaping neutrinos lead the shock to stall and turn into an accretion shock soon after bounce. In the hot postshock region, electrons are less degenerate and positrons appear and are captured on neutrons, leading to a rise of the $\bar{v}_{e}$ luminosity. In addition, in the PNS and in the postshock region, neutrinos and antineutrinos of all flavors are emitted by thermal processes.

7 The neutrinosphere is the effective 'decoupling' surface of neutrinos where the optical depth $\tau_{v}$ of the supernova matter is $2 / 3$. Its position depends strongly on neutrino energy. 
The simple $\bar{Y}_{e}(\rho)$ parameterization discussed in section 4.1 is not adequate to capture these effects and, in principle, a full neutrino energy-dependent radiation-hydrodynamics treatment would be needed for accurately capturing postbounce neutrino effects. Such a treatment may be added in future versions of GR1D. In the present version of GR1D, we approximate postbounce neutrino transport by a gray (energy-averaged) neutrino leakage scheme augmented with a simple prescription for neutrino heating in the postshock region. This approach captures the most important qualitative aspects of the postbounce evolution well and, as we demonstrate in section 6.2, is sufficiently quantitatively accurate to make reliable predictions of the time of $\mathrm{BH}$ formation and the maximum PNS mass in failing core-collapse supernovae.

Our implementation in GR1D combines elements of the neutrino leakage schemes of Ruffert et al [64] and of Rosswog and Liebendörfer [65]. We consider three neutrino species, $v_{e}, \bar{v}_{e}$ and $v_{x}$. In the latter, we lump together $\mu$ and $\tau$ neutrinos and antineutrinos since they interact only by neutral-current processes in the core-collapse context and have very similar cross sections. The mean (energy-averaged) optical depth is

$$
\tau_{v_{i}}(r)=\int_{r}^{\infty} \kappa_{t}\left(v_{i}\right) X \mathrm{~d} r,
$$

where $\kappa_{t}\left(v_{i}\right)$ is the mean transport opacity equal to the sum of absorptive and scattering opacities $^{8}$ for neutrino species $v_{i}$. We follow [64] in the calculation of $\kappa_{t}\left(v_{i}\right)$ and of the approximate neutrino degeneracy parameters $\left(\eta_{v_{i}}=\mu_{v_{i}} / T\right)$. We consider opacity contributions from neutrino scattering on neutrons, protons and heavy nuclei and absorption of neutrinos (antineutrinos) on neutrons (protons). For heavy-lepton neutrinos that are never degenerate, we set $\eta_{v_{x}}=0$. $\eta_{v_{e}}$ is known (1) in $\beta$-equilibrium where $\eta_{v_{e}}^{\mathrm{eq}}=\eta_{e}+\eta_{p}-\eta_{n}$ (where we assume that the chemical potentials include rest mass terms) and (2) in the free streaming limit, where $\eta_{v_{e}}^{\text {stream }}=0$. Furthermore, $\eta_{\bar{v}_{e}}^{\mathrm{eq}}=-\eta_{\nu_{e}}^{\mathrm{eq}}$. In between the two regimes, the neutrino distribution function cannot be derived from first principles and neutrino transport is necessary for a correct estimate of $\eta_{v_{e}}$ and $\eta_{\bar{v}_{e}}$. As an approximation, we interpolate between (1) and (2) using the optical depth:

$$
\eta_{v_{i}}=\eta_{v_{i}}^{\mathrm{eq}}\left(1-\mathrm{e}^{-\tau_{v_{i}}\left(\eta_{v_{i}}\right)}\right) \text {. }
$$

Note that $\tau_{v_{i}}$ depends on $\eta_{\nu_{i}}$ and vice versa. Hence, we iterate their calculation until convergence is reached ${ }^{9}$.

Knowing $\tau_{v_{i}}$ and $\eta_{v_{i}}$, we use the leakage scheme of [65] to calculate the neutrino emission rates for the capture processes $p+e^{-} \rightarrow v_{e}+n$ and $e^{+}+n \rightarrow \bar{v}_{e}+p$ and thermal emission via electron-positron annihilation and plasmon decay to $v \bar{v}$ pairs. We modify the scheme of [65] in the following ways: (i) we use the interpolated $\eta_{v_{i}}$ from above instead of the equilibrium values suggested in [65], (ii) we increase their diffusion time scale $t_{v_{i}}^{\text {diff }}$ by a factor of 2 to obtain more reasonable neutrino luminosity predictions and (iii) for simplicity, we use the analytic thermal emissivities from [64]. Following [65], we then interpolate the effective volumetric energy loss $Q_{\text {eff }}^{\text {leak }}\left(\mathrm{erg} / \mathrm{cm}^{3} / \mathrm{s}\right)$ and the effective number loss $R_{\text {eff }}^{\text {leak }}\left(\# / \mathrm{cm}^{3} / \mathrm{s}\right)$ between the limits of diffusive emission (subscript 'diff') and free emission (subscript 'loc') using

$$
\chi_{\mathrm{eff}, v_{i}}^{\text {leak }}=\chi_{\mathrm{loc}, v_{i}}^{\text {leak }} /\left(1+\chi_{\mathrm{loc}, v_{i}}^{\text {leak }} / \chi_{\mathrm{diff}, v_{i}}^{\text {leak }}\right)
$$

where $\chi=Q$ for energy loss and $\chi=R$ for number loss (see [65] for definitions and details). We define the neutrino luminosity seen by an observer at rest at radius $r$ in the coordinate frame

8 Note that the opacities for the neutrino number and neutrino energy transport differ. Hence, the optical depths for number and energy transport must be computed separately [64]. We neglect this subtlety and use the optical depths for energy transport throughout GR1D.

9 Initially we choose $\kappa_{v_{i}}(r)=10^{-5} \mathrm{~cm}^{-1}$, determine $\tau_{v_{i}}$ through (23) and iterate (24). For all subsequent times we use the previously determined value of $\tau_{v_{i}}$ as a starting point, and convergence (fractional difference in $\kappa_{\nu_{i}}<10^{-10}$ ) is typically reached after three iterations. 
by summing up the effective energy emission rates from each zone interior to $r$, transforming from the fluid rest frame (FRF) to the coordinate frame $(\mathrm{CF})$ and applying the redshift (see appendix B for details):

$L_{v_{i}}^{\mathrm{CF}}(r)=4 \pi \int_{0}^{r}\left[\frac{\alpha\left(r^{\prime}\right)}{\alpha(r)}\right] Q_{\mathrm{eff}, v_{i}}\left(r^{\prime}\right)\left[\alpha\left(r^{\prime}\right) W\left(r^{\prime}\right)\left(1+v\left(r^{\prime}\right)\right)\right] X\left(r^{\prime}\right) r^{\prime 2} \mathrm{~d} r^{\prime}$.

For an observer at rest at $r=\infty(\alpha(\infty)=1)$,

$L_{v_{i}}(\infty)=4 \pi \int_{0}^{\infty} \alpha\left(r^{\prime}\right) Q_{\mathrm{eff}, v_{i}}\left(r^{\prime}\right)\left[\alpha\left(r^{\prime}\right) W\left(r^{\prime}\right)\left(1+v\left(r^{\prime}\right)\right)\right] X\left(r^{\prime}\right) r^{\prime 2} \mathrm{~d} r^{\prime}$.

It is useful to note the neutrino luminosity as seen by an observer at rest in the fluid rest frame at radius $r$ :

$$
L_{v_{i}}^{\mathrm{FRF}}(r)=\frac{L_{v_{i}}^{\mathrm{CF}}(r)}{\alpha(r) W(r)(1+v(r))},
$$

where the denominator transforms the luminosity from the frame of an observer at rest in the coordinate frame (26) to the fluid rest frame.

4.2.1. Neutrino heating. In addition to the above leakage scheme, we include a parameterized heating scheme to mimic neutrino absorption in the postshock region. Heating occurs at intermediate to low optical depths where neutrinos begin to decouple from matter and a net energy transfer from neutrinos to the fluid is possible (see, e.g., [89]). The dominant heating processes are the charged-current capture reactions of $v_{e}$ on neutrons and $\bar{v}_{e}$ on protons. We take the absorption cross sections from [65],

$$
\begin{aligned}
\sigma_{\text {heat }, v_{e}} & =\frac{\left(1+3 g_{A}^{2}\right)}{4} \sigma_{0} \frac{\left\langle\epsilon^{2}\right\rangle_{\nu_{e}}^{\mathrm{ns}}}{\left(m_{e} c^{2}\right)^{2}}\left\langle 1-f_{e^{-}}\right\rangle, \\
\sigma_{\text {heat }, \bar{v}_{e}} & =\frac{\left(1+3 g_{A}^{2}\right)}{4} \sigma_{0} \frac{\left\langle\epsilon^{2}\right\rangle_{\bar{v}_{e}}^{\mathrm{ns}}}{\left(m_{e} c^{2}\right)^{2}}\left\langle 1-f_{e^{+}}\right\rangle,
\end{aligned}
$$

where $\sigma_{0}$ is a reference weak-interaction cross section equal to $1.76 \times 10^{-44} \mathrm{~cm}^{2}, g_{A} \sim-1.25$ and the Fermi blocking factors $\left\langle 1-f_{i}\right\rangle$ are defined analogously to $[64,65]$. In the postshock region the positron blocking term is negligible but the electron blocking term can be significant around the time of bounce. Following [89], we set the mean squared neutrino energy to $\left\langle\epsilon^{2}\right\rangle_{v_{i}}^{\mathrm{ns}}=T\left(\tau_{v_{i}}=\frac{2}{3}\right)^{2} \mathcal{F}_{5}\left(\eta_{v_{i}}^{\mathrm{ns}}\right) / \mathcal{F}_{3}\left(\eta_{v_{i}}^{\mathrm{ns}}\right)$, where $T\left(\tau_{v_{i}}=\frac{2}{3}\right)$ is the temperature at the neutrinosphere of species $i$, superscript ns denotes neutrinospheric values and $\mathcal{F}_{n}(\eta)=\int_{0}^{\infty} \frac{x^{n} \mathrm{~d} x}{\exp (x-\eta)+1}$ is the $n$th Fermi integral (we approximate Fermi integrals via the formulas given in [90]).

Given the neutrino luminosity $L_{v_{i}}^{\mathrm{FRF}}(r)$ obtained from the leakage scheme (28), we write the local neutrino heating rate in units of $\operatorname{erg~cm}^{-3} \mathrm{~s}^{-1}$ as

$$
Q_{v_{i}}^{\text {heat }}(r)=f_{\text {heat }} \frac{L_{v_{i}}^{\mathrm{FRF}}(r)}{4 \pi r^{2}} \sigma_{\text {heat }, v_{i}} \frac{\rho}{m_{u}} X_{i}\left(\frac{1}{F_{v_{i}}}\right) \mathrm{e}^{-2 \tau_{v_{i}}},
$$

where $m_{u}$ is the atomic mass unit and the mass fraction $X_{i}=X_{n}$ in the case of $v_{e}$ absorption and $X_{i}=X_{p}$ for $\bar{v}_{e}$ s. $\left\langle 1 / F_{v_{i}}\right\rangle$ is the mean inverse flux factor describing the degree of forwardpeaking of the radiation field (e.g. [44, 89]; $\left\langle 1 / F_{v_{i}}\right\rangle$ is 1 for free streaming and diverges at high optical depth). We estimate $\left\langle 1 / F_{v_{i}}\right\rangle$ by the interpolation $\left\langle 1 / F_{\nu_{i}}(\tau)\right\rangle=4.275 \tau+1.15$, which reproduces the predicted values of 4 at the neutrinosphere [89] and levels off at a value of 1.15 at a low optical depth in the outer postshock region. We choose the latter value instead 
of 1 because (a) the radiation field becomes fully forward peaked only outside the shock (e.g. [44]), and (b) the linear interpolation in $\tau$ drops off too quickly compared to full simulations [44], and hence the higher floor value to compensate. Finally, we introduce the attenuation factor $\mathrm{e}^{-2 \tau_{v_{i}}}$ to cut off heating near and below the neutrinosphere and the scaling factor $f_{\text {heat }}$ to allow for an ad hoc increase of the heating rate. Once the heating rate for a computational cell is computed, we reduce the outgoing luminosity by the deposited power for overall energy conservation. In the coordinate frame (26) now becomes

$L_{v_{i}}^{\mathrm{CF}}(r)=4 \pi \int_{0}^{r}\left[\frac{\alpha\left(r^{\prime}\right)}{\alpha(r)}\right]\left[Q_{\mathrm{eff}, v_{i}}\left(r^{\prime}\right)-Q_{v_{i}}^{\text {heat }}\left(r^{\prime}\right)\right]\left[\alpha\left(r^{\prime}\right) W\left(r^{\prime}\right)\left(1+v\left(r^{\prime}\right)\right)\right] X\left(r^{\prime}\right) r^{\prime 2} \mathrm{~d} r^{\prime}$.

Along with the energy deposition goes a change in $Y_{e}$ which can be written as

$$
R_{Y_{e}}^{\text {heat }}=\frac{Q_{v_{e}}^{\text {heat }}}{\left\langle\epsilon_{v_{e}}^{\text {ns }}\right\rangle}-\frac{Q_{\bar{v}_{e}}^{\text {heat }}}{\left\langle\epsilon_{\bar{v}_{e}}^{\mathrm{ns}}\right\rangle},
$$

where we approximate the mean neutrino energies based on their neutrinospheric values as $\left\langle\epsilon_{v_{i}}^{\mathrm{ns}}\right\rangle=T\left(\tau_{v_{e}}=\frac{2}{3}\right) \mathcal{F}_{5}\left(\eta_{v_{i}}^{\mathrm{ns}}\right) / \mathcal{F}_{4}\left(\eta_{v_{i}}^{\mathrm{ns}}\right)[65]$

To caution the reader, we point out that the simple gray heating scheme presented above is not self-consistent and cannot replace a radiation transport treatment that allows emission and absorption to balance. While we find that the combination of gray leakage/heating reproduces the overall qualitative dynamical features observed in postbounce radiation-hydrodynamic simulations, quantitative aspects are not captured as well. This is true in particular in highly dynamical situations shortly after bounce when we observe an unphysical rise of the electron fraction due to heating in the lower postshock region.

We couple the neutrino leakage/heating scheme with the GR hydrodynamics in GR1D through source/sink terms on the RHS of the GR hydrodynamics equations in MoL. Neutrinomatter interactions occur in the fluid rest frame where the total energy and number changes are given by

$$
Q_{\mathrm{E}}^{0}=Q_{\text {total }}^{\text {heat }}-Q_{\text {eff,total }}^{\text {leak }}, \quad R_{Y_{e}}^{0}=R_{\text {total }}^{\text {heat }}+R_{\text {eff,total }}^{\text {leak }},
$$

where $Q_{\text {total }}^{\text {heat }}$ and $Q_{\text {eff,total }}^{\text {leak }}$ are always positive or zero and $R_{\text {total }}^{\text {heat }}$ and $R_{\text {eff,total }}^{\text {leak }}$ may be positive or negative. Following $[40,56]$, transforming these terms to the coordinate frame via the methods laid out in appendix A, we obtain the neutrino heating/cooling and deleptonization source/sink terms for the RHS in the MoL integration:

$$
R_{Y_{e}}^{v}=\alpha X R_{Y_{e}}^{0} \quad Q_{S^{r}}^{\nu, E}=\alpha v W Q_{\mathrm{E}}^{0} \quad Q_{\tau}^{\nu, E}=\alpha W Q_{\mathrm{E}}^{0} .
$$

\subsection{Neutrino pressure}

Electron neutrinos above trapping density in the inner core during the final phases of collapse and in the postbounce PNS contribute to both the pressure and the specific energy density (with relative importance of up to $\sim 10 \%$ around core bounce [91]). We neglect neutrino contributions to pressure and energy below $\rho_{\text {trap }}$ where they are small, but otherwise follow [63] and assume electron neutrinos and antineutrinos to be a perfect Fermi gas. The pressure is then given by

$$
P_{v}=\frac{4 \pi}{3(h c)^{3}} T^{4}\left[F_{3}\left(\eta_{v}\right)+F_{3}\left(-\eta_{v}\right)\right]
$$

where $\eta_{v}=\mu_{v} / T$ and $\mu_{v}=\mu_{e}-\mu_{n}+\mu_{p}$, where the chemical potentials include rest mass contributions. $F_{3}$ is the third Fermi integral which we approximate following [92]. The specific internal energy of a relativistic Fermi gas of neutrinos is simply $\epsilon_{v}=3 P_{v} / \rho$. 
Table 1. Initial conditions for two relativistic shocktube problems as presented in [93].

\begin{tabular}{ll|ll}
\hline \multicolumn{2}{c|}{$\mathrm{P} 1$} & \multicolumn{2}{c}{$\mathrm{P} 2$} \\
\hline$r<0.5$ & $r>0.5$ & $r<0.5$ & $r>0.5$ \\
$\rho=10$ & $\rho=1$ & $\rho=1$ & $\rho=1$ \\
$P=13.33$ & $P=0$ & $P=10^{3}$ & $P=0.01$ \\
$v=0$ & $v=0$ & $v=0$ & $v=0$ \\
\hline
\end{tabular}

We treat neutrinos and fluid separately from each other and treat momentum transfer between the neutrino radiation field and the fluid approximately using the radial gradient of the neutrino pressure as suggested by [63]. We couple this radiation stress into GR1D's MoL integration of the GR momentum $\left(S^{r}\right)$ and energy $(\tau)$ equations via source terms (see appendix A for a derivation; we neglect rotational effects in these source terms):

$$
Q_{S^{r}}^{v, \mathrm{M}}=-\alpha W \frac{\partial P_{v}}{\partial r}, \quad Q_{\tau}^{v, \mathrm{M}}=-\alpha W v \frac{\partial P_{v}}{\partial r} .
$$

In addition to the force on the fluid due to the neutrino pressure gradient, we take into account the energy and 'pressure' of the neutrino radiation field by adding $P_{v}$ and $\epsilon_{v}$ through the terms $\tau_{m}^{\nu}$ and $\tau_{\Phi}^{v}$ in (4) and equations (5) and (15). These contributions are derived by modifying the stress-energy tensor:

$$
T^{\alpha \beta}=\rho\left[1+\left(\epsilon+\epsilon_{v}\right)+\left(\frac{P+P_{v}}{\rho}\right)\right] u^{\alpha} u^{\beta}+g^{\alpha \beta}\left(P+P_{\nu}\right) ;
$$

$\tau_{m}^{\nu}$ and $\tau_{\Phi}^{\nu}$ are then given by [35]

$$
\begin{aligned}
& \tau_{m}^{\nu}=\rho W^{2}\left(\epsilon_{v}+P_{\nu} / \rho\right)-P_{v}=\left(4 W^{2}-1\right) P_{\nu}, \\
& \tau_{\Phi}^{v}=\rho W^{2} v^{2}\left(\epsilon_{v}+P_{\nu} / \rho\right)+P_{v}=\left(4 W^{2} v^{2}+1\right) P_{\nu} .
\end{aligned}
$$

We note that if rotation is included, $v^{2}$ in (40) is replaced with $v^{2}+\frac{2}{3} v_{\varphi}^{2}$.

\section{Code tests}

In the following, we provide results from a set of standard and stringent relativistic hydrodynamics code tests for which analytic results exist. These involve two planar shocktube problems in section 5.1, the spherical Sedov blast wave problem in section 5.2 and the Oppenheimer-Snyder collapse in section 5.3. Finally, in section 5.4, we present results from a collapse simulation of an $n=3$ polytrope and demonstrate convergence of the hydrodynamics scheme in GR1D. With this selection, we test a broad range of aspects of potential problems to be addressed with GR1D: special relativistic effects, geometrical effects and fully generalrelativistic collapse dynamics.

\subsection{Relativistic shocktube}

We assume flat space and planar geometry and perform the two relativistic shocktube tests proposed by [93]. We use a $\Gamma$-law EOS with $\Gamma=5 / 3$ and a grid of length 1 with a cell spacing of $\mathrm{d} x=0.001$. The starting values of the density, pressure and velocity are summarized in table 1 . The left panel of figure 1 shows the exact results for velocity, density and pressure of the mildly relativistic problem 1 at $t=0.4$. Superposed are the numerical results obtained with GR1D that reproduce the exact results nearly perfectly. Problem 2 is a more stringent test 

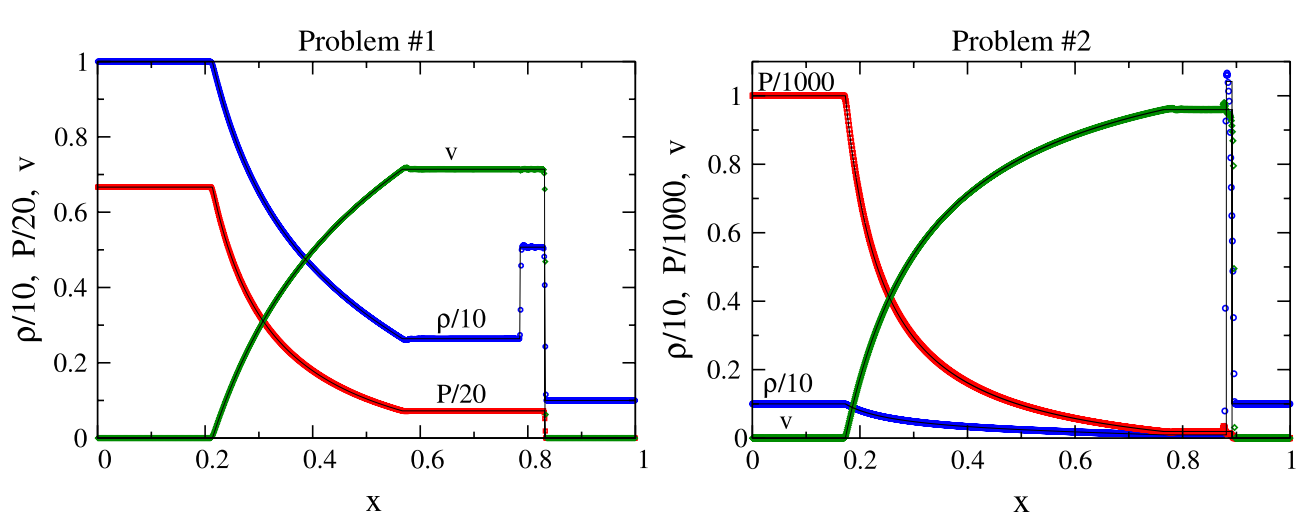

Figure 1. Relativistic shocktube simulations: initial conditions taken from [93] and provided in table 1 . The pressure, density and velocity are shown at $t=0.4$ for problem 1 (left panel) and problem 2 (right panel). For reference, in both figures the pressure is denoted by boxes (red online), density by circles (blue online) and velocity by diamonds (green online). The analytic solution is denoted by the solid line. Both problems were run with a Courant factor of 0.5 and third-order Runge-Kutta integration.

and involves Lorentz factors of up to 6 in the forward propagating shock and a very thin shell of trailing matter. As shown in the right panel of section 1, GR1D reproduces the exact solution at $t=0.4$ very well almost everywhere, but fails to completely resolve the thin shell of relativistic matter. This is most likely due to the rather diffusive nature of the HLLE Riemann solver employed in GR1D (see, e.g., [91, 94] for comparable results obtained with a nominally more accurate scheme). In an attempt to obtain results closer to the analytic solution we use third-order Runge-Kutta time integration for this test case. These deviations are not worrying since the shocks obtain in stellar collapse are much less relativistic than those of problem 2 . If GR1D were to be applied to ultrarelativistic outflows (e.g. in a GRB), a more precise treatment of the Riemann problem would likely be necessary.

\subsection{Sedov blast wave}

The above shocktube tests demonstrated the ability of GR1D to capture shocks and solve the special-relativistic hydrodynamic equations in planar geometry. Here we go back to Newtonian hydrodynamics and test instead spherical hydrodynamics with Sedov's blast wave problem [95]. For a comparison with a large number of hydrodynamics codes, we use the initial conditions of [96]. The grid setup is in spherical geometry with (dimensionless) $r_{\max }=10$ and $N=400$ cells which corresponds to the maximum mesh refinement level used in [96]. We deposit a constant specific internal energy into a sphere of radius $r=0.0875$, corresponding to a total (dimensionless) energy of $E_{o}=10^{5}$, into a background medium of (dimensionless) $\rho_{0}=1$. We set the background energy density to an insignificant amount and use a $\Gamma$-law EOS with $\Gamma=5 / 3$. Figure 2 depicts the comparison of our numerical solution with the exact result for density, velocity and pressure at $t=0.1$ normalized in such a way that the value of all variables at the shock is 1 . GR1D performs very well in the region behind the shock and provides an adequate, though not perfect, solution near the shock.

In addition to the Newtonian Sedov blast wave problem, we have also considered its relativistic variant discussed in [97]. These authors used 17 levels of adaptive mesh refinement (AMR) and we find that the lack of AMR in GR1D makes it computationally impossible to 


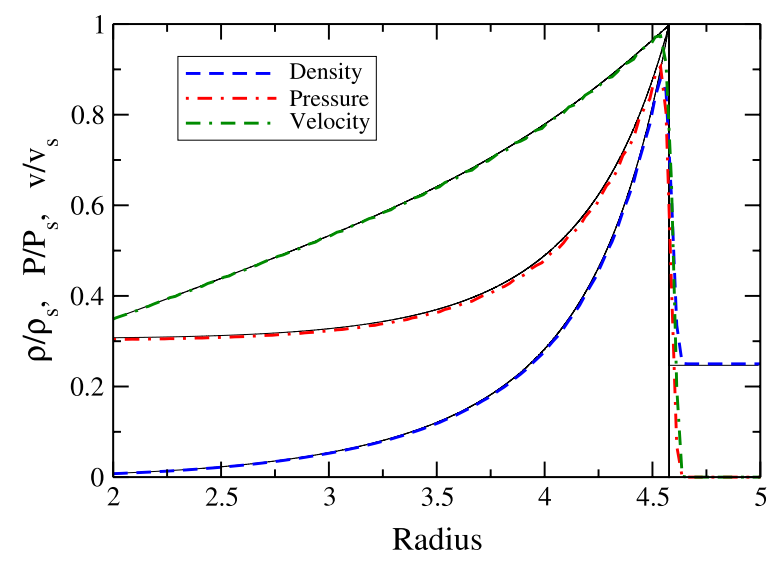

Figure 2. The Sedov blast wave problem and the exact solution at $t=0.1$. Shown are the numerical results with the exact solution underlying the various curves of density, pressure and velocity. Both the exact solution and the numerical result are normalized to the analytic value at the shock. $\rho_{\mathrm{s}}=4, P_{s}=252.255$ and $v_{s}=13.757$.

adequately resolve the relativistic Sedov problem. This, however, is not a problem for the application of GR1D to the stellar collapse problem, since the shocks appearing there are only mildly relativistic.

\subsection{Oppenheimer-Snyder collapse}

For the final test problem for which an exact solution exists, we perform a simulation of the Oppenheimer-Snyder collapse (OSC) [98] of a constant-density sphere of pressureless $(P=0)$ dust. The exact solution of OSC in RGPS spacetime has been laid out by [99, 100]. We choose $M=M_{\odot}, R_{\star}=10 M_{\odot}$. We perform the OSC test with the standard version of GR1D described in section 2 of this paper and do not make special adjustments for the code to operate with $P=0$. Hence, we set the pressure to a small, but non-zero value, using a polytropic EOS with $K=10^{-20}$ and $\Gamma=5 / 3$. In the artificial atmosphere outside the dust ball, we set the density to $1 \mathrm{~g} \mathrm{~cm}^{-3}$. We use 9000 equidistant zones to model OSC with GR1D.

In figure 3, we compare numerical and exact density and lapse profiles of OSC at $t=30,35,40,43$ and $60 M_{\odot}$. Following [34], we normalize the central density to the value at $t=0$. The overall agreement is excellent. However, we note two slight deviations: (1), near the origin, we observe a small build up of material. This is present also in the OSC test of [34] and probably due to diverging terms near the origin. We do not note this effect in our stellar collapse calculations, most likely because of the stabilizing effect of the large pressure in the PNS. (2), at late times $\left(t>50 M_{\odot}\right)$, the numerical $\alpha$ decreases more slowly than its exact counterpart and begins to deviate significantly at $\alpha(r=0) \lesssim 0.001$. We attribute this to numerical inaccuracies developing due (a) to the metric coefficient $X$ becoming singular as $R_{\star} \rightarrow 2 M_{\odot}$, (b) to the extreme density gradient developing at the surface at late times, and (c) to the fact that we use the standard version of GR1D without special adjustments for the OSC problem (as, e.g., made by [34]).

\subsection{Hybrid core collapse: convergence}

In this section, we present simulations of nonrotating core collapse and present the proof of convergence for GR1D. We utilize the hybrid EOS described in section 3.1, taking $\Gamma_{1}=1.28$, 

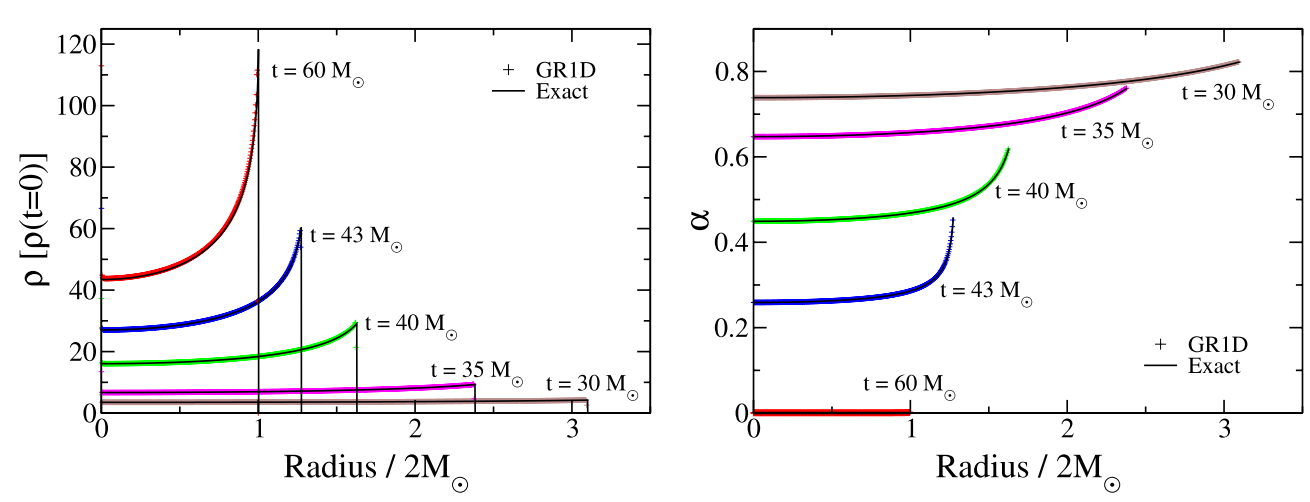

Figure 3. Oppenheimer-Snyder collapse of a pressureless dust ball. Shown are the numerical (plus symbols) and exact (solid lines) density (left panel) and lapse (right panel) profiles for various times. The density is normalized to the density at $t=0$. The simulation uses 9000 equally spaced grid points across the domain of $20 M_{\odot}$. Initially one solar mass is distributed with constant density in a sphere of radius $10 M_{\odot}$. For clarity, we show only every third data point.

$\Gamma_{2}=2.5, \Gamma_{\text {th }}=1.5$ and $K=4.935 \times 10^{14}$ [cgs]. Following [91], we use as initial data an $n=3$ polytrope with a central density of $\rho_{c}=5 \times 10^{10} \mathrm{~g} \mathrm{~cm}^{-3}$ and a $K$ value as above and initially zero radial velocity. We simulate the evolution with GR1D for equally spaced grids of three different resolutions $\left(N_{\text {zones }}=500,1500\right.$ and 4500) to test the self-convergence of the code. The self-convergence factor at convergence order $n$ of a quantity $q$ is given by

$$
Q=\frac{q_{1}-q_{2}}{q_{2}-q_{3}}=\frac{\left(\mathrm{d} x_{1}\right)^{n}-\left(\mathrm{d} x_{2}\right)^{n}}{\left(\mathrm{~d} x_{2}\right)^{n}-\left(\mathrm{d} x_{3}\right)^{n}}
$$

where $q_{i}$ is the numerical result from the simulation with the corresponding resolution and $\mathrm{d} x$ is the zone width. For this convergence test, $\mathrm{d} x_{1}=3 \mathrm{~d} x_{2}=9 \mathrm{~d} x_{3}$. In the lower panel of figure 4, we show the self-convergence of $M_{\text {grav }}$ at $t=-3.3 \mathrm{~ms}$ (before bounce) as well as at $t=16.6 \mathrm{~ms}$ and $t=26.6 \mathrm{~ms}$ after bounce.

We generally see the expected second-order convergence $(Q=9)$ in smooth parts of the flow, but note several interesting features: (1) before bounce (red online, dot-dashed curve) and near $120 \mathrm{~km}$ where the convergence spikes, the velocity is peaking, causing a reduction in convergence. (2) During the postbounce phase, convergence in the shocked region drops to first order; this is characteristic of HRSC schemes in the presence of shocks. (3) Finally, during the postbounce phase for $r<20 \mathrm{~km}$, the steepness of the density gradient at the PNS surface and the coarseness of the grid lead to local non-convergence. We note that the lowest resolution used here is $\mathrm{d} x \sim 2 \mathrm{~km}$ and that deviations in the density profile compared to higher resolution simulations can be seen in the top panel of figure 4 .

\section{Sample results for a $40 M_{\odot}$ star}

In the following simulations we use the single-star, nonrotating, $M_{\text {ZAMS }}=40 M_{\odot}$, solarmetallicity presupernova model of Woosley and Weaver [88] (model s40WW95 hereafter). This model has an iron core mass of $1.98 M_{\odot}$. We set up a grid of 1000 zones that is logarithmically spaced from $r=20 \mathrm{~km}$ outward, extending to a radius of $1.15 \times 10^{5} \mathrm{~km}$ where the density drops to $200 \mathrm{~g} \mathrm{~cm}^{-3}$. There is $14.7 M_{\odot}$ of baryonic material within this density cutoff. Inside $r=20 \mathrm{~km}$, we use an equidistant grid with a spacing of $100 \mathrm{~m}$. Such high resolution is necessary to resolve steep gradients at the PNS surface at late times 


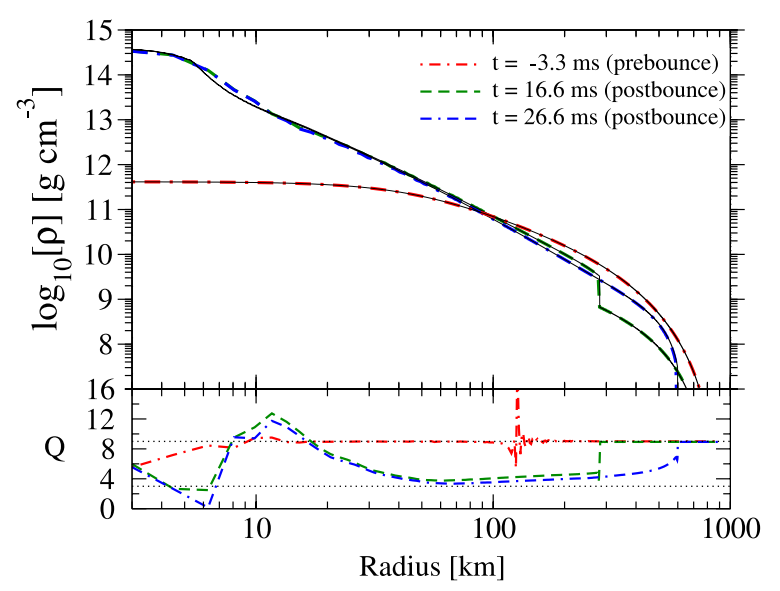

Figure 4. Radial density profiles and self-convergence for core collapse using the hybrid EOS. Top: density profiles of the core collapse for various times including the prebounce phase, and after the shock has propagated through $\sim 300$ and $600 \mathrm{~km}$. We show the low-resolution profile (segmented lines) as well as the high-resolution profile (solid lines) for comparison. Bottom: self-convergence of the enclosed gravitational mass, $m(r)$. The dotted lines at $Q=3$ and 9 denote the expected values for first- and second-order convergence.

( $t \gtrsim 0.5 \mathrm{~s}$ ). Near the origin, we increase the zone size gradually to $\sim 700 \mathrm{~m}$ for improved stability but for rotating runs we find it necessary to maintain the fine grid spacing all the way to the origin to capture the correct angular velocity profile.

\subsection{Rotating core collapse and black hole formation in a $40 M_{\odot}$ star using the hybrid EOS}

To show the effects of including rotation and to further demonstrate the use and usefulness of the hybrid EOS (see section 3.1) for exploratory studies, we perform a set of collapse simulations to black hole formation. We set $\Gamma_{1}=1.30, \Gamma_{2}=2.5, \Gamma_{\text {th }}=1.34$ and impose rotation according to the rotation law (see, e.g., $[77,80]$ )

$$
\Omega(r)=\xi \frac{\pi}{10}\left[1+\left(\frac{r}{A}\right)^{2}\right]^{-1} \operatorname{rad~s}^{-1},
$$

where we vary $\xi$ from 0 to 5 and $A$ is a parameter governing the degree of differential rotation. We choose $A=1000 \mathrm{~km}$ which leads to roughly uniform rotation within the inner core as predicted by stellar evolutionary calculations (e.g. [74]). As an additional test of GR1D, we show in the lower part of the left panel of figure 5 the relative error in total angular momentum and gravitational mass in the most rapidly spinning simulation. GR1D conserves angular momentum to better than one part in $10^{4}$ and $M_{\text {grav }}$ to one part in $10^{6}$ until the onset of $\mathrm{BH}$ formation when the resolution becomes insufficient to fully resolve the huge gradients in the collapsing PNS.

We show in the top part of figure 5 the evolution of the central density in the simulated models. Due to the choice of $\Gamma_{1}$, rotation has little influence on the prebounce dynamics [52]. The hybrid EOS qualitatively captures the stiffening of the EOS at nuclear density that leads to core bounce. Owing to the small value of $\Gamma_{\text {th }}$, the shock stalls soon after bounce and accretion on the PNS continues. Slowly spinning models accrete rapidly and collapse to a $\mathrm{BH}$ after only $200 \mathrm{~ms}$. Centrifugal support becomes dynamically relevant in more rapidly spinning cases, decreasing the accretion rate and delaying $\mathrm{BH}$ formation. The right panel of figure 5 depicts 

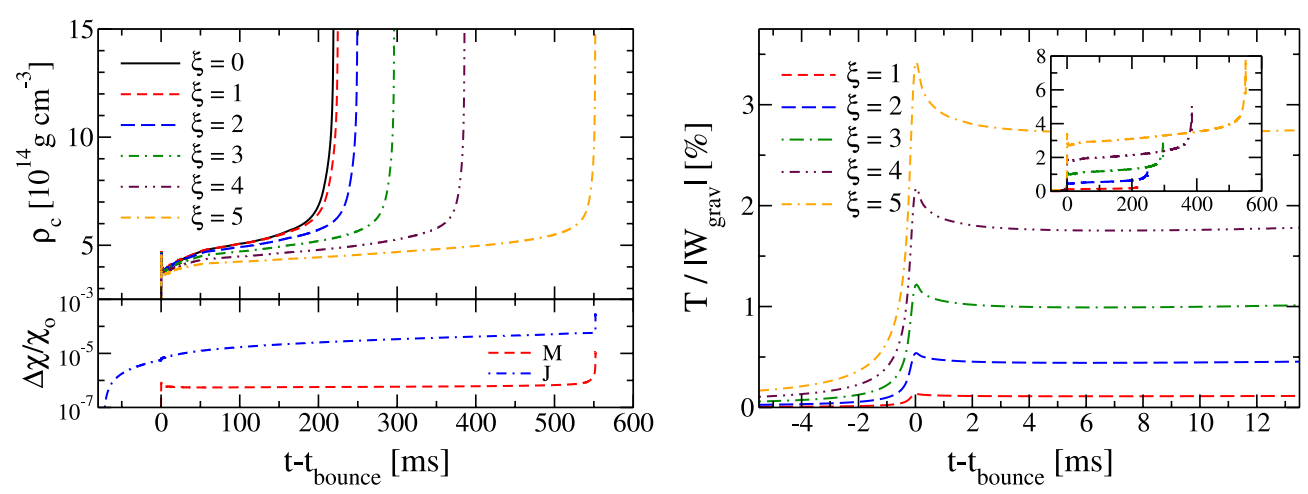

Figure 5. Black hole formation with rotation and the hybrid EOS. Left panel: central densities for various initial angular velocities (top) and fractional error of the conserved quantities $M_{\text {grav }}$ and $J$ (bottom). Right panel: $T /\left|W_{\text {grav }}\right|$ near bounce and (inset) over the entire simulation. $\Omega(r)$ is set through (42).

the evolution of the rotation parameter $T /\left|W_{\text {grav }}\right|$. Its systematics are very similar to what has been observed in multi-D simulations (e.g. $[53,77,101]) . T /\left|W_{\text {grav }}\right|$ reaches a local maximum at bounce, and then decreases as the PNS reaches its postbounce quasi-equilibrium. New and not shown before is the evolution of $T /\left|W_{\text {grav }}\right|$ near $\mathrm{BH}$ formation. $T /\left|W_{\text {grav }}\right|$ increases only slowly after bounce (note that, in a calculation with neutrino transport or leakage, the postbounce $T /\left|W_{\text {grav }}\right|$ would increase faster [77]), but near BH formation grows nearly exponentially during PNS collapse. Rotation, in particular when it is strongly differential, can increase the maximum mass of the accreting PNS (e.g. [102]). We find ${ }^{10} \mathrm{BH}$ birth masses of 1.89-1.97 $M_{\odot}$ for the set of rotating hybrid-EOS models considered here. This increase in the maximum mass is modest, primarily because our PNS cores are rather uniformly spinning (in agreement with $[53,77])$. We point out that our present treatment does not consider angular momentum redistribution by multi-dimensional effects or effective viscosity which may be present in realistic systems (see, e.g., [54, 76] and references therein).

Finally, we note that for the nonrotating $(\xi=0)$ model, the evolution with GR1D continues until a central value of the lapse function of $3 \times 10^{-10}$ and a maximum value of $\sqrt{g_{r r}}=X$ of $\sim 21.1$. These are excellent values in comparison to previous studies on $\mathrm{BH}$ formation in RGPS [30, 100]. In the rotating case, the evolution terminates somewhat earlier due primarily to numerical issues near the origin at very large $v_{\varphi}$.

\subsection{Nonrotating collapse and black hole formation with neutrino leakage/heating in a $40 M_{\odot}$ star}

In this section we show example results employing GR1D's leakage/heating scheme and finite-temperature EOS. We use the s40WW95 progenitor and the $\operatorname{LS} 180 \operatorname{EOS}^{11}, Y_{e}(\rho)$ parameterization prebounce, our standard leakage/heating scheme after bounce and no rotation. We show results for both $f_{\text {heat }}=0$ (losses only) and $f_{\text {heat }}=1$. In figure 6 , we compare the shock radii of these two runs and neutrino luminosities of the $f_{\text {heat }}=1$ run

${ }^{10}$ In RGPS, a coordinate singularity develops at $R=2 M$ upon $\mathrm{BH}$ formation. We define here the $\mathrm{BH}$ mass to be $M_{\text {grav }}$ inside the radius that corresponds to the maximum $X$. This is an approximation and is subject to errors due to our finite resolution grid.

${ }^{11}$ The lower bound on our EOS tables is $1000 \mathrm{~g} \mathrm{~cm}^{-3}$, and we bring the outer boundary into $\rho=2000 \mathrm{~g} \mathrm{~cm}^{-3}$ for this example. 

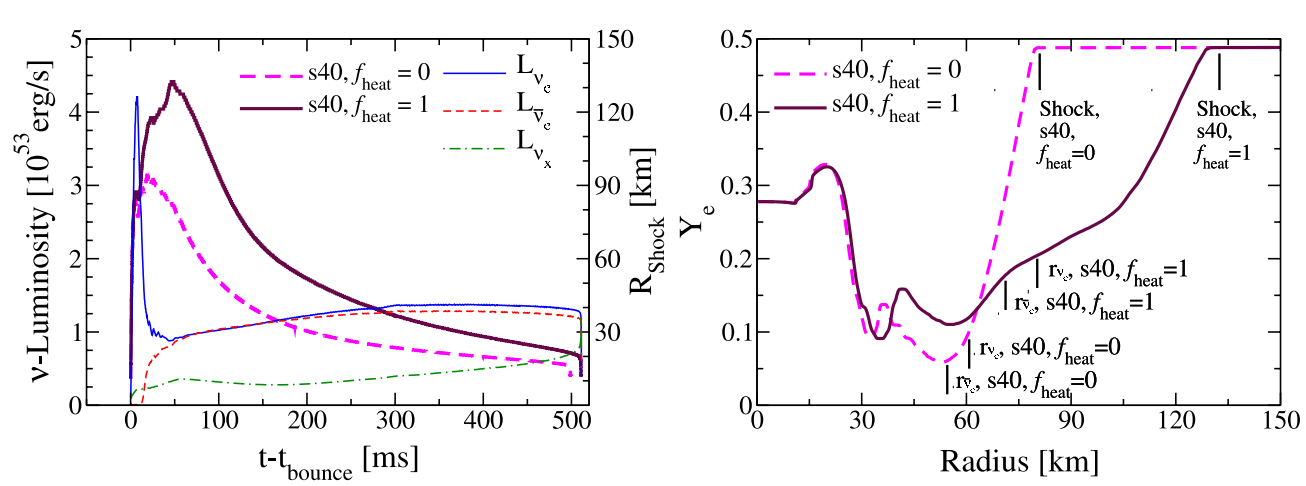

Figure 6. Left panel: shock radius (thick line, right ordinate) and neutrino luminosities (thin lines, left ordinate) as a function of postbounce time in a nonrotating leakage+heating $\left(f_{\text {heat }}=1\right)$ simulation with the $40 M_{\odot}$ model of [88] run with the LS180 EOS. Shown also is the shock radius evolution (dashed thick lines) in a simulation without heating $f_{\text {heat }}=0$. Right panel: $Y_{e}$ profiles of both simulations at $50 \mathrm{~ms}$ after bounce, corresponding to the maximum shock radius of the $f_{\text {heat }}=1$ simulation. Shock radii, electron and anti-electron neutrino neutrinospheres are marked for both the $f_{\text {heat }}=1$ and $f_{\text {heat }}=0$ simulations.

(left panel) as well as the $Y_{e}$ radial profiles at $50 \mathrm{~ms}$ after bounce (right panel). We note that the total luminosity is $L_{v_{e}}+L_{\bar{v}_{e}}+4 L_{v_{\mu}}$ and is corrected for redshift through (32) with $r=\infty$, but, nevertheless, is somewhat higher (up to $\sim 20 \%$ ) than predicted by full Boltzmann radiation-hydrodynamics calculations using the same progenitor [103, 104]. The time until $\mathrm{BH}$ formation in the case of $f_{\text {heat }}=1$ is $t_{\mathrm{BH}}=511 \mathrm{~ms}$ and the baryonic mass inside the shock of the last stable configuration is $2.25 M_{\odot}$. We compare this with two other studies of $\mathrm{BH}$ formation in $1 \mathrm{D}$ with the same progenitor model and EOS, but with two different implementations of GR Boltzmann neutrino transport. These studies are Fischer et al [103] who found $t_{\mathrm{BH}}=435.5 \mathrm{~ms}$ and $2.196 M_{\odot}$ and Sumiyoshi et al [104] who found $t_{\mathrm{BH}}=560 \mathrm{~ms}$ and $2.1 M_{\odot}$. Our result is very close to these more accurate studies which gives us confidence in the robustness of the heating/leakage scheme in GR1D.

The right panel of figure 6 depicts the $Y_{e}$ profiles at $50 \mathrm{~ms}$ after bounce. The characteristic trough in $Y_{e}$ behind the shock is captured by our leakage/heating scheme, but we find that our simple heating scheme converts too many of the postshock neutrons back to protons at early times, leading to too high values of $Y_{e}$ in the lower postshock region between $\sim 30-60 \mathrm{~km}$.

To conclude this section, we note that, due to the computational efficiency of our scheme, each of our simulations took only $\sim 6 \mathrm{CPU}$ hours from iron core collapse through BH formation on one core of an Intel Xeon X5550 (Nehalem) machine.

\section{Summary and concluding remarks}

In this paper, we have presented the details of our new open-source Eulerian 1.5D GR hydrodynamics code GR1D. GR1D is intended primarily for the simulation of stellar collapse to neutron stars and black holes and, for the first time in the 1D GR context, includes an approximate way of accounting for stellar rotation consistent with that used in state-of-the-art calculations of stellar evolution (e.g. [74]). Using this scheme, we have presented rotating long-term postbounce simulations toward black hole formation using a $40 M_{\odot}$ supernova progenitor model and showed how the simple analytic hybrid EOS can be used to capture many qualitative aspects of this phenomenon. 
As we have demonstrated in this paper, GR1D performs well in standard tests and, despite its simplified neutrino leakage/heating scheme, still yields overall results in the case of failing core-collapse supernovae and black hole formation that measure up qualitatively and to some extent also quantitatively to those obtained with full Boltzmann neutrino transport in 1D Lagrangian codes [103, 104].

Many 1D GR (radiation)-hydrodynamics formulations have been presented in the past $\sim 50$ years. Yet, there is presently no open-source 1D GR stellar collapse code available to the broader community. The primary motivation driving the development of GR1D is the need for such an open-source code that may be used as a codebase, benchmark and testbed for improved modeling technology to be included in multi-D GR codes addressing core-collapse supernova explosions, but also failing core-collapse supernovae, black hole formation and the post-merger evolution of binary neutron-star and neutron-star-black hole coalescence. Equipped with an approximate neutrino-leakage scheme to capture the key effects associated with neutrino heating and cooling, the version of GR1D discussed in this paper is a solid starting point for the next generation of astrophysically relevant multi-D GR simulations.

The current limitations of GR1D due to its gray leakage and simplified heating scheme are obvious. We will continue to develop and improve GR1D and intend to include as a next step energy-dependent radiation transport in the multi-group flux-limited diffusion approximation (MGFLD) and/or in the isotropic diffusion source approximation (IDSA, [105]).

\section{Acknowledgments}

We thank the Niels Bohr International Academy for hosting the Microphysics in Computational Relativistics Astrophysics (MICRA) workshop in August 2009 at which much of the work presented here was inspired. It is a pleasure to thank J-M Ibánẽz for helpful advice, for providing the original version of the code of Romero et al and for furnishing a copy of Romero's dissertation. We are indebted to M Duez for very valuable help with the derivation of the neutrino source terms. We are furthermore happy to acknowledge helpful exchanges with W D Arnett, A Burrows, P Cerdá-Durán, H Dimmelmeier, T Fischer, E Gourgoulhon, I Hawke, J Lattimer, L Lehner, M Liebendörfer, E Livne, C Meakin, S Noble, A Perego, C Pethick, E S Phinney, E Schnetter, S Scheidgger, Y Sekiguchi and S Teukolsky. This work is supported by the National Science Foundation under grant numbers AST-0855535 and OCI-0905046. EOC is supported in part through a post-graduate fellowship from the Natural Sciences and Engineering Research Council of Canada (NSERC) and NASA ATP grant NNX07AH06G. We wish to thank Chris Mach for support of our group servers at TAPIR on which much of the code development and testing was carried out. Results presented in this paper were obtained through computations on the NSF Teragrid under grant TG-MCA02N014, on machines of the Louisiana Optical Network Initiative under grant LONI_NUMREL04 and at the National Energy Research Scientific Computing Center (NERSC), which is supported by the Office of Science of the US Department of Energy under contract DE-AC03-76SF00098.

\section{Appendix A. Evolution equation derivation}

In this appendix we derive the evolution equations for the conserved variables $D, D Y_{e}, S^{r}, S_{\phi}$ and $\tau$ used in GR1D and presented in section 2.2 and section 2.3. GR1D uses the spherically symmetric metric $g_{\mu \nu}=\operatorname{diag}\left(-\alpha^{2}, X^{2}, r^{2}, r^{2} \sin ^{2} \theta\right)$ with $\alpha=\exp (\Phi(r, t))$ where $\Phi(r, t)$ is defined through (5), $X=\left(1-\frac{2 m(r, t)}{r}\right)^{-1 / 2}$ where $m(r, t)$ is the enclosed gravitational mass at the coordinate radius $r$. We assume the matter to be a perfect fluid described by a mass current 
Table A1. Connection coefficients.

\begin{tabular}{|c|c|}
\hline$\Gamma^{t}{ }_{t t}=\partial_{t} \phi(r, t)$ & $\Gamma_{\theta \theta}^{r}=-\frac{r}{X^{2}}$ \\
\hline$\Gamma_{t r}^{t}=\partial_{r} \phi(r, t)$ & $\Gamma_{\phi \phi}^{r}=-\frac{r \sin ^{2} \theta}{X^{2}}$ \\
\hline$\Gamma_{r r}^{t}=\alpha^{-2} \frac{X^{4}}{r} \partial_{t} m(r, t)$ & $\Gamma_{r \theta}^{\theta}=\frac{1}{r}$ \\
\hline$\Gamma_{t t}^{r}=\frac{\alpha^{2}}{X^{2}} \partial_{r} \phi(r, t)$ & $\Gamma_{\phi \phi}^{\theta}=-\sin \theta \cos \theta$ \\
\hline$\Gamma_{t r}^{r}=\frac{X^{2}}{r} \partial_{t} m(r, t)$ & $\Gamma_{r \phi}^{\phi}=\frac{1}{r}$ \\
\hline$\Gamma_{r r}^{r}=\frac{X^{2}}{r}\left(\partial_{r} m(r, t)-\frac{m(r, t)}{r}\right)$ & $\Gamma_{\theta \phi}^{\phi}=\frac{\cos \theta}{\sin \theta}$ \\
\hline
\end{tabular}

density of $J^{\mu}=\rho u^{\mu}$ and a stress-energy tensor $T^{\mu \nu}=\rho h u^{\mu} u^{\nu}+g^{\mu \nu} P$ where $\rho$ is the rest mass density, $P$ is the fluid pressure, $h=1+\epsilon+P / \rho$ is the specific enthalpy with $\epsilon$ being the specific internal energy, $u^{\mu}=(W / \alpha, W v / X, 0,0)$ is the fluid 4-velocity (without taking into account rotation) with $W=1 / \sqrt{1-v^{2}}$ is the Lorentz factor and $v$ is the physical radial velocity.

While evaluating the covariant derivative of the stress-energy tensor and matter current density, we make use of the following formulas:

$$
\nabla_{\mu} J^{\mu}=\frac{1}{\sqrt{-g}}\left(\sqrt{-g} J^{\mu}\right)_{, \mu}
$$

and

$$
\nabla_{\mu} T^{\mu \nu}=\frac{1}{\sqrt{-g}}\left(\sqrt{-g} T^{\mu \nu}\right)_{, \mu}+\Gamma_{\alpha \mu}^{\nu} T^{\mu \alpha},
$$

where $\sqrt{-g}=\alpha X r^{2}$ is the determinant of the metric and $\Gamma^{\nu}{ }_{\alpha \mu}$ are Christoffel symbols and are defined through derivatives of the metric:

$$
\Gamma_{\alpha \mu}^{\nu}=\frac{1}{2} g^{\nu \beta}\left(g_{\mu \beta, \alpha}+g_{\alpha \beta, \mu}-g_{\alpha \mu, \beta}\right) .
$$

For our metric, all non-zero Christoffels are given in table A1, $\Gamma^{\nu}{ }_{\alpha \mu}$ is symmetric in the last two indices and duplicates are omitted.

It is useful to note the following derivatives needed in the derivation of the evolution equations:

$$
\begin{aligned}
& \partial_{r} \Phi=X^{2}\left[\frac{m}{r^{2}}+4 \pi r\left(P+\rho h W^{2} v^{2}\right)\right], \\
& \partial_{r} X=X^{3}\left[\frac{\partial_{r} m}{r}-\frac{m}{r^{2}}\right], \\
& \partial_{t} X=X^{3} \frac{\partial_{t} m}{r} \\
& \partial_{r} m=4 \pi r^{2}\left(\rho h W^{2}-P\right), \\
& \partial_{t} m=-4 \pi r^{2} \frac{\alpha \rho h W^{2} v}{X} .
\end{aligned}
$$

\section{A.1. Source terms}

The evolution equations follow from $\nabla_{\mu} J^{\mu}=0$ and $\nabla_{\mu} T^{\mu \nu}=0$. Since we treat neutrinos through a leakage scheme, we add in neutrino source terms explicitly to the RHS of these equations. The neutrino physics of GR1D occurs in the rest frame of the fluid; in this frame 
the energy and lepton rates are calculated with the neutrino leakage scheme, $Q_{E}^{0}$ and $R_{Y_{e}}^{0}$ are given in (34). Momentum exchange in the fluid rest frame is taken into account approximately via $Q_{M}^{0}=-\frac{\partial P_{v}}{\partial r}$ where the gradient is evaluated numerically in the coordinate frame. This introduces a slight inconsistency, since in a full radiation-transport treatment the momentum transfer is computed fully locally via the second angular moment of the local neutrino radiation intensity [58].

By writing the evolution equations in the comoving orthonormal frame of the fluid (fluid rest frame, FRF) with 4-velocity $\vec{u}=(1,0,0,0)_{\mathrm{FRF}}$ and unit radial normal $\vec{n}=(0,1,0,0)_{\mathrm{FRF}}$ and expressing them as frame-independent tensor equations we can derive expressions for the evolution equations in any frame. For the lepton fraction,

$$
\begin{aligned}
& \partial_{t}\left(\rho Y_{e}\right)=R_{Y_{e}}^{0}, \\
& \partial_{t}\left(\rho Y_{e} u^{t}\right)=R_{Y_{e}}^{0}, \\
& \partial_{\mu}\left(\rho Y_{e} u^{\mu}\right)=R_{Y_{e}}^{0}, \\
& \nabla_{\mu}\left(\rho Y_{e} u^{\mu}\right)=R_{Y_{e}}^{0} .
\end{aligned}
$$

We write the energy and momentum source terms in the fluid rest frame as a 4-vector, $\vec{q}=\left(Q_{E}^{0}, Q_{M}^{0}, 0,0\right)_{\mathrm{FRF}}$, or in the frame-independent notation, $Q_{E}^{0} \vec{u}+Q_{M}^{0} \vec{n}$. In the fluid rest frame, the evolution equations for energy and momentum become

$$
\partial_{t} T^{t t}=Q_{E}^{0}=q^{t}
$$

and

$$
\partial_{t} T^{t r}=Q_{M}^{0}=q^{r}
$$

or in the frame-independent tensor notation,

$$
\nabla_{\mu} T^{\mu \nu}=q^{v}
$$

For the evolution equations, we must transform $\vec{q}$ from the fluid rest frame to the coordinate frame $(\mathrm{CF})$ of GR1D. In a general frame $\vec{n}$ is a vector that is both (i) normalized and (ii) orthogonal to $\vec{u}$. In the CF of GR1D, where $\vec{u}$ is the 4-velocity, these two conditions (along with the assumption of spherical symmetry) on $\vec{n}$ give $\vec{n}=(W v / \alpha, W / X, 0,0)_{\mathrm{CF}} \cdot \vec{q}$ in the $\mathrm{CF}$ then becomes $\vec{q}=\left(\frac{W}{\alpha}\left(Q_{E}^{0}+v Q_{M}^{0}\right), \frac{W}{X}\left(v Q_{E}^{0}+Q_{M}^{0}\right), 0,0\right)_{\mathrm{CF}}$. This can also be derived via a Lorentz transformation. In principle, non-zero rotation will give rise to source terms for the $\phi$-momentum evolution through $q^{\phi}$ and modify the radial source terms $q^{r}$. In consideration of the significant approximations already present in both our neutrino leakage scheme and our treatment of rotation, we neglect the influence of rotation on the source terms. This is justified as long as $v_{\varphi} \ll c$.

\section{A.2. GR1D evolution equations}

In the coordinate frame of GR1D where $u^{\mu}=(W / \alpha, W v / X, 0,0)$, the continuity equation, $\nabla_{\mu} J^{\mu}=0$, gives the evolution of the rest mass density:

$$
\begin{aligned}
& \nabla_{\mu}\left(\rho u^{\mu}\right)=0 \\
& \frac{1}{\sqrt{-g}}\left[\partial_{t}\left(\sqrt{-g} \frac{\rho W}{\alpha}\right)+\partial_{r}\left(\sqrt{-g} \frac{\rho W v}{X}\right)\right]=0 \\
& \partial_{t}(D)+\frac{1}{r^{2}} \partial_{r}\left(\frac{\alpha r^{2}}{X} D v\right)=0 .
\end{aligned}
$$


The evolution of the electron fraction $Y_{e}$ follows a similar derivation but contains a source term from the neutrino leakage scheme. In the coordinate frame of GR1D (A.9) becomes

$$
\begin{aligned}
& \nabla_{\mu}\left(\rho Y_{e} u^{\mu}\right)=R_{Y_{e}}^{0} \\
& \frac{1}{\sqrt{-g}}\left[\partial_{t}\left(\sqrt{-g} \frac{\rho W Y_{e}}{\alpha}\right)+\partial_{r}\left(\sqrt{-g} \frac{\rho W Y_{e} v}{X}\right)\right]=R_{Y_{e}}^{0}, \\
& \frac{1}{\alpha X}\left[\partial_{t}\left(X \rho W Y_{e}\right)+\frac{1}{r^{2}} \partial_{r}\left(\frac{\alpha r^{2}}{X} X \rho W Y_{e} v\right)\right]=R_{Y_{e}}^{0}, \\
& \partial_{t}\left(D Y_{e}\right)+\frac{1}{r^{2}} \partial_{r}\left(\frac{\alpha r^{2}}{X} D Y_{e} v\right)=\alpha X R_{Y_{e}}^{0} .
\end{aligned}
$$

The momentum evolution equation for GR1D is obtained by evaluating (A.12) with $v=r$, $\nabla_{\mu} T^{\mu r}=q^{r}$,

$$
\begin{aligned}
& \left(\sqrt{-g} T^{\mu r}\right)_{, \mu}=\sqrt{-g} q^{r}-\sqrt{-g} \Gamma_{\nu \mu}^{r} T^{\mu \nu}, \\
& \partial_{t}\left(\rho h W^{2} v\right)+\frac{1}{r^{2}} \partial_{r}\left(\frac{\alpha r^{2}}{X}\left(\rho h W^{2} v^{2}+P\right)\right)=\alpha X q^{r}-\alpha X\left(\Gamma_{v t}^{r} T^{t v}+\Gamma_{v r}^{r} T^{r v}\right. \\
& \left.+\Gamma_{v \phi}^{r} T^{\phi v}+\Gamma_{v \theta}^{r} T^{\theta v}\right) \\
& \partial_{t}\left(S^{r}\right)+\frac{1}{r^{2}} \partial_{r}\left(\frac{\alpha r^{2}}{X}\left(S^{r} v+P\right)\right)=\alpha X q^{r}-\alpha X\left(\Gamma_{t t}^{r} T^{t t}+\Gamma_{r t}^{r} T^{t r}\right. \\
& \left.+\Gamma_{t r}^{r} T^{r t}+\Gamma_{r r}^{r} T^{r r}+\Gamma_{\phi \phi}^{r} T^{\phi \phi}+\Gamma_{\theta \theta}^{r} T^{\theta \theta}\right), \\
& \partial_{t}\left(S^{r}\right)+\frac{1}{r^{2}} \partial_{r}\left(\frac{\alpha r^{2}}{X}\left(S^{r} v+P\right)\right)=-\alpha X\left[2 \frac{X}{r} \frac{\rho h W^{2} v}{\alpha X} \partial_{t} m\right. \\
& +\frac{X^{2}}{r}\left(\partial_{r} m-\frac{m}{r}\right)\left(\frac{\rho h W^{2} v^{2}+P}{X^{2}}\right) \\
& \left.-\frac{2 P}{X^{2} r}+\frac{\alpha^{2}}{X^{2}} \frac{\rho h W^{2}-P}{\alpha^{2}} \partial_{r} \Phi\right]+\alpha X q^{r} \\
& \partial_{t}\left(S^{r}\right)+\frac{1}{r^{2}} \partial_{r}\left[\frac{\alpha r^{2}}{X}\left(S^{r} v+P\right)\right]=\alpha X\left[\left(S^{r} v-\tau-D\right)\left(8 \pi r P+\frac{m}{r^{2}}\right)\right. \\
& \left.+\frac{P m}{r^{2}}+\frac{2 P}{X^{2} r}\right]+\alpha W\left(v Q_{E}^{0}+Q_{M}^{0}\right)
\end{aligned}
$$

where in the last step we have reorganized the source terms to the form of [34] using the derivatives defined in (A.4)-(A.8). If non-zero, $u^{\phi}=W v_{\varphi} / r$ leads to an additional term $\left(\alpha \rho h W^{2} v_{\varphi}^{2} \sin (\theta)^{2} / X r\right)$ arising through $\Gamma_{\phi \phi}^{r} T^{\phi \phi}$ on the RHS of (A.15); averaging this term over the spherical shell gives $2 / 3 \alpha \rho h W^{2} v_{\varphi}^{2} / X r$. When rotation is included, the evolution equation for $S_{\phi}=\rho h W^{2} v_{\varphi} r$ is

$\nabla_{\mu} T_{\phi}^{\mu}=0$

$\left(\sqrt{-g} T_{\phi}^{\mu}\right)_{, \mu}=\sqrt{-g} \Gamma_{\phi \mu}^{v} T_{v}^{\mu}$

$\partial_{t}\left(\alpha X r^{2} g_{\phi \phi} T^{t \phi}\right)+\partial_{r}\left(\alpha X r^{2} g_{\phi \phi} T^{r \phi}\right)=\sqrt{-g}\left(\Gamma_{\phi \phi}^{r} T_{r}^{\phi}+\Gamma_{\phi r}^{\phi} T_{\phi}^{r}\right)$,

$\partial_{t}\left(X \rho h W^{2} v_{\varphi} r\right)+\frac{1}{r^{2}} \partial_{r}\left(\frac{\alpha r^{2}}{X} \rho h W^{2} v_{\varphi} r v X\right)=0$, 


$$
\begin{aligned}
& \partial_{t}\left(S_{\phi}\right)+\frac{1}{r^{2}} \partial_{r}\left(\frac{\alpha r^{2}}{X} S_{\phi} v\right)=\frac{\rho h W^{2} v_{\varphi} r}{X}\left(-\partial_{t} X-\frac{\alpha v}{X} \partial_{r} X\right), \\
& \partial_{t}\left(S_{\phi}\right)+\frac{1}{r^{2}} \partial_{r}\left(\frac{\alpha r^{2}}{X} S_{\phi} v\right)=\alpha \rho h W^{2} v_{\varphi} v X\left(4 \pi r^{2} P+\frac{m}{r}\right) .
\end{aligned}
$$

The energy evolution equation for GR1D is derived by taking $v=t$ in (A.12):

$$
\begin{aligned}
& \nabla_{\mu} T^{\mu t}=q^{t}, \\
& \left(\sqrt{-g} T^{\mu t}\right)_{, \mu}=\sqrt{-g} q^{t}-\sqrt{-g} \Gamma_{\nu \mu}^{t} T^{\mu \nu} \\
& \partial_{t}\left(\frac{X}{\alpha}\left(\rho h W^{2}-P\right)\right)+\frac{1}{r^{2}} \partial_{r}\left(\frac{\alpha r^{2}}{X} \rho h W^{2} v \frac{X}{\alpha}\right)=\alpha X q^{t}-\alpha X\left(\Gamma_{t \mu}^{t} T^{\mu t}+\Gamma_{r \mu}^{t} T^{\mu r}\right) \\
& \frac{X}{\alpha}\left[\partial_{t}(\tau+D)+\frac{1}{r^{2}} \partial_{r}\left(\frac{\alpha r^{2}}{X} S^{r}\right)\right]=\alpha X q^{t}-\alpha X\left(\Gamma_{t t}^{t} T^{t t}+2 \Gamma_{t r}^{t} T^{r t}+\Gamma_{r r}^{t} T^{r r}\right) \\
& \quad-\left(\rho h W^{2}-P\right) \partial_{t}\left(\frac{X}{\alpha}\right)-\frac{\alpha \rho h W^{2} v}{X} \partial_{r}\left(\frac{X}{\alpha}\right) \\
& \partial_{t}(\tau+D)+\frac{1}{r^{2}} \partial_{r}\left(\frac{\alpha r^{2}}{X} S^{r}\right)=\alpha^{2} q^{t}, \\
& \partial_{t}(\tau)+\frac{1}{r^{2}} \partial_{r}\left(\frac{\alpha r^{2}}{X}\left(S^{r}-D v\right)\right)=\alpha W\left(Q_{E}^{0}+v Q_{M}^{0}\right)
\end{aligned}
$$

where in the last step we use the continuity equation (A.13) to subtract out the evolution of the rest mass density, obtaining the evolution equation for $\tau$. A non-zero $u^{\phi}$ does not contribute source terms to this evolution equation.

\section{Appendix B. Neutrino luminosities}

The luminosity computed from the neutrino leakage scheme is derived in the rest frame of the fluid. We require knowledge of the neutrino luminosity as measured by an observer at rest in the coordinate frame to determine (i) the luminosity measured by an observer at rest at infinity and (ii) the luminosity in the fluid rest frame at some other coordinate radius for our neutrino heating scheme. We derive these relationships by assuming that the neutrinos are emitted radially in the fluid rest frame with energy $E^{\mathrm{FRF}}$.

In the fluid rest frame (FRF), the 4-momentum of the (massless) neutrino is $p^{a}=$ $\left(E^{\mathrm{FRF}}, E^{\mathrm{FRF}}, 0,0\right)_{\mathrm{FRF}}$. We use the orthonormal tetrad in appendix A.1, in the fluid frame, $\vec{u}=\vec{e}_{0}=(1,0,0,0)_{\mathrm{FRF}}$ and $\vec{n}=\vec{e}_{1}=(0,1,0,0)_{\mathrm{FRF}}$, in the coordinate frame (CF), $u^{\beta}=e_{0}^{\beta}=(W / \alpha, W v / X, 0,0)_{\mathrm{CF}}$ and $n^{\beta}=e_{1}^{\beta}=(W v / \alpha, W / X, 0,0)_{\mathrm{CF}}$. In this we have neglected rotational effects which will be small for $v_{\varphi} \ll c$. Transforming $p^{a}$ to the coordinate basis of GR1D,

$$
p^{\beta}=p^{a} e_{a}^{\beta}=E^{\mathrm{FRF}}\left(\frac{W}{\alpha}(1+v), \frac{W}{X}(1+v), 0,0\right)_{\mathrm{CF}} .
$$

An observer at rest in the coordinate frame $\left(U^{\alpha}=(1,0,0,0)_{\mathrm{CF}}\right)$ then sees the neutrino with energy:

$E^{\mathrm{CF}}=-\vec{p} \cdot \vec{U}=-g_{\alpha \beta} p^{\beta} U^{\alpha}=\alpha^{2} E^{\mathrm{FRF}} \frac{W}{\alpha}(1+v)=\alpha W(1+v) E^{\mathrm{FRF}}$. 
Noting that (see [106], equation 25.25) for massless particles emitted from rest at $r$ and observed by an observer at rest at $r^{\prime}, \lambda(r)\left|g_{00}(r)\right|^{-1 / 2}=\lambda\left(r^{\prime}\right)\left|g_{00}\left(r^{\prime}\right)\right|^{-1 / 2}$ implies

$$
\frac{E^{\mathrm{CF}}\left(r^{\prime}\right)}{E^{\mathrm{CF}}(r)}=\frac{\lambda_{r}}{\lambda_{r^{\prime}}}=\frac{\left|g_{00}(r)\right|^{1 / 2}}{\left|g_{00}\left(r^{\prime}\right)\right|^{1 / 2}}=\frac{\alpha(r)}{\alpha\left(r^{\prime}\right)} ;
$$

this is the redshift formula for particles leaving a gravitational well.

\section{References}

[1] Bethe H A 1990 Rev. Mod. Phys. 62801

[2] Liebendörfer M, Rampp M, Janka H-T and Mezzacappa A 2005 Astrophys. J. 620840

[3] Buras R, Janka H-T, Rampp M and Kifonidis K 2006 Astron. Astrophys. 457281

[4] Marek A and Janka H-T 2009 Astrophys. J. 694664

[5] Janka H-T, Langanke K, Marek A, Martínez-Pinedo G and Müller B 2007 Phys. Rep. 44238

[6] Burrows A, Dessart L, Ott C D and Livne E 2007 Phys. Rep. 44223

[7] Bruenn S W, Mezzacappa A, Hix W R, Blondin J M, Marronetti P, Messer O E B, Dirk C J and Yoshida S 2009 Mechanisms of Core-Collapse Supernovae and Simulation Results from the CHIMERA Code (AIP Phys. Conf. Ser. vol 1111) ed G Giobbi, A Tornambe, G Raimondo, M Limongi, L A Antonelli, N Menci and E Brocato $\mathrm{p} 593$

[8] Ott C D 2009 Class. Quantum Grav. 26204015

[9] Zhang W, Woosley S E and Heger A 2008 Astrophys. J. 679639

[10] Woosley S E 1993 Astrophys. J. 405273

[11] Woosley S E and Bloom J S 2006 Annu. Rev. Astron. Astrophys. 44507

[12] May M M and White R H 1966 Phys. Rev. 1411232

[13] Misner C W and Sharp D H 1964 Phys. Rev. 136571

[14] Von Neumann J and Richtmyer R D 1950 J. Appl. Phys. 21232

[15] van Riper K A 1979 Astrophys. J. 232558

[16] Baumgarte T W, Shapiro S L and Teukolsky S A 1995 Astrophys. J. 443717

[17] Swesty F D 1995 Astrophys. J. 445811

[18] Liebendörfer M, Rosswog S and Thielemann F-K 2002 Astrophys. J. Suppl. Ser. 141229

[19] Miralles J A, Ibanez J M, Marti J M and Perez A 1991 Astron. Astrophys. Suppl. 90 283-99

[20] Schinder P J, Bludman S A and Piran T 1988 Phys. Rev. D. 372722

[21] Wilson J R 1971 Astrophys. J. 163209

[22] Bruenn S W 1985 Astrophys. J. Suppl. Ser. 58771

[23] Baron E, Cooperstein J and Kahana S 1985 Phys. Rev. Lett. 55126

[24] Baron E, Myra E S, Cooperstein J and van den Horn L J 1989 Astrophys. J. 339978

[25] Liebendörfer M, Messer O E B, Mezzacappa A, Bruenn S W, Cardall C Y and Thielemann F-K 2004 Astrophys. J. Suppl. Ser. 150263

[26] Wilson J R 1979 A numerical method for relativistic hydrodynamics Sources of Gravitational Radiation ed L L Smarr (Cambridge: Cambridge University Press) p 423

[27] Shapiro S L and Teukolsky S A 1979 Astrophys. J. Lett. 234 L177

[28] Shapiro S L and Teukolsky S A 1980 Astrophys. J. 235199

[29] Mezzacappa A and Matzner R A 1989 Astrophys. J. 343853

[30] Gourgoulhon E 1991 Astron. Astrophys. 252651

[31] Martí J M, Ibáñez J M and Miralles J A 1990 Astron. Astrophys. 235535

[32] Yamada S 1997 Astrophys. J. 475720

[33] Martí J M, Ibáñez J M and Miralles J A 1991 Phys. Rev. D. 433794

[34] Romero J V, Ibáñez J M, Martí J M and Miralles J A 1996 Astrophys. J. 462839

[35] Noble S C 2003 PhD Thesis University of Britsh Columbia (arXiv:gr-qc/0310116)

[36] Yamada S, Janka H-T and Suzuki H 1999 Astron. Astrophys. 344533

[37] Sumiyoshi K, Yamada S, Suzuki H, Shen H, Chiba S and Toki H 2005 Astrophys. J. 629922

[38] Gourgoulhon E and Haensel P 1993 Astron. Astrophys. 271187

[39] Romero J V, Miralles J M, Ibáñez J A and Pons J A General relativistic collapse of hot stellar cores Some Topics on General Relativity and Gravitational Radiation ed J A Miralles, J A Morales and D Saez (Paris: Editions Frontières) p 289 
[40] Pons J A, Miralles J A and Ibáñez J M 1997 General relativistic neutrino transport Some Topics on General Relativity and Gravitational Radiationed J A Miralles, J A Morales and D Saez (Paris: Editions Frontières) p 293

[41] Thompson T A, Burrows A and Pinto P A 2003 Astrophys. J. 592434

[42] Burrows A, Livne E, Dessart L, Ott C D and Murphy J 2006 Astrophys. J. 640878

[43] Burrows A, Dessart L, Livne E, Ott C D and Murphy J 2007 Astrophys. J. 664416

[44] Ott C D, Burrows A, Dessart L and Livne E 2008 Astrophys. J. 6851069

[45] Swesty F D and Myra E S 2009 Astrophys. J. Suppl. Ser. 1811

[46] Burrows A, Livne E, Dessart L, Ott C D and Murphy J 2007 Astrophys. J. 655416

[47] Isenberg J A 2008 Int. J. Mod. Phys. D 17265

[48] Dimmelmeier H, Font J A and Müller E 2002 Astron. Astrophys. 393523

[49] Dimmelmeier H, Novak J, Font J A, Ibáñez J M and Müller E 2005 Phys. Rev. D. 71064023

[50] Shibata M and Sekiguchi Y 2004 Phys. Rev. D 69084024

[51] Shibata M and Sekiguchi Y I 2005 Phys. Rev. D. 71024014

[52] Dimmelmeier H, Ott C D, Janka H-T, Marek A and Müller E 2007 Phys. Rev. Lett. 98251101

[53] Dimmelmeier H, Ott C D, Marek A and Janka H-T 2008 Phys. Rev. D. 78064056

[54] Ott C D, Dimmelmeier H, Marek A, Janka H-T, Hawke I, Zink B and Schnetter E 2007 Phys. Rev. Lett. 98261101

[55] Ott C D, Dimmelmeier H, Marek A, Janka H-T, Zink B, Hawke I and Schnetter E 2007 Class. Quantum Grav. 24139

[56] Müller B 2009 Multi-dimensional relativistic simulations of core-collapse supernovae with energy-dependent neutrino transport PhD Thesis Technische Universität München, München, Germany

[57] Buras R, Rampp M, Janka H-T and Kifonidis K 2006 Astron. Astrophys. 4471049

[58] Müller B, Janka H-T and Dimmelmeier H 2010 Astrophys. J. Suppl. Ser. (submitted) (arXiv:1001.4841 [astro-ph])

[59] URL http://www.whiskycode.org/ Whisky, EU Network GR Hydrodynamics Code.

[60] Lattimer J M and Swesty F D 1991 Nucl. Phys. A 535331

[61] Shen H, Toki H, Oyamatsu K and Sumiyoshi K 1998 Nucl. Phys. A 637435 (http://user.numazuct.ac.jp/ sumi/eos)

[62] Shen H, Toki H, Oyamatsu K and Sumiyoshi K 1998 Prog. Theor. Phys. 1001013

[63] Liebendörfer M 2005 Astrophys. J. 6331042

[64] Ruffert M, Janka H-T and Schaefer G 1996 Astron. Astrophys. 311532

[65] Rosswog S and Liebendörfer M 2003 Mon. Not. R. Astron. Soc. 342673

[66] Banyuls F, Font J A, Ibáñez J M, Martí J M and Miralles J A 1997 Astrophys. J. 476221

[67] Font J A, Miller M, Suen W-M and Tobias M 2000 Phys. Rev. D. 61044011

[68] Font J A 2008 Liv. Rev. Rel. 117

[69] Hyman J M 1976 The method of lines solution of partial differential equations Technical Report COO-3077139 (ERDA Mathematics and Computing Laboratory, Courant Institute of Mathematical Sciences, New York University)

[70] Colella P and Woodward P R 1984 J. Comput. Phys. 54 174-201

[71] Leer B J van 1977 J. Comput. Phys. 23276

[72] Einfeldt B 1988 On Godunov type methods for the Euler equations with a general equation of state Shock Tubes and Waves: Proc. of the 16th Int. Symp. (Aachen, Germany, 26-31 July 1987) A89-12876 03-34 (Weinheim, Germany: VCH Verlagsgesellschaft mbH) pp 671-6

[73] Endal A S and Sofia S 1978 Astrophys. J. 220279

[74] Heger A, Langer N and Woosley S E 2000 Astrophys. J. 528368

[75] Hirschi R, Meynet G and Maeder A 2004 Astron. Astrophys. 425 649-70

[76] Thompson T A, Quataert E and Burrows A 2005 Astrophys. J. 620861

[77] Ott C D, Burrows A, Thompson T A, Livne E and Walder R 2006 Astrophys. J. Suppl. Ser. 164130

[78] Cook G B, Shapiro S L and Teukolsky S A 1992 Astrophys. J. 398 203-23

[79] Janka H-T, Zwerger T and Mönchmeyer R 1993 Astron. Astrophys. 268360

[80] Zwerger T and Müller E 1997 Astron. Astrophys. 320209

[81] Read J S, Lackey B D, Owen B J and Friedman J L 2009 Phys. Rev. D. 79124032

[82] Shapiro L S and Teukolsky S A 1983 Black Holes, White Dwarfs and Neutron Stars (New York: Wiley)

[83] Lattimer J M, Pethick C J, Ravenhall D G and Lamb D Q 1985 Nucl. Phys. A 432646

[84] Timmes F X and Arnett D 1999 Astrophys. J. Suppl. Ser. 125277

[85] Rampp M and Janka H-T 2002 Astron. Astrophys. 396361

[86] Timmes F X and Swesty F D 2000 Astrophys. J. Suppl. Ser. 126501 
[87] Steffen M 1990 Astron. Astrophys. 239443

[88] Woosley S E and Weaver T A 1995 Astrophys. J. Supp. Ser. 101181

[89] Janka H-T 2001 Astron. Astrophys. 368527

[90] Takahashi K, El Eid M F and Hillebrandt W 1978 Astron. Astrophys. 67185

[91] Ott C D 2006 Stellar iron core collapse in 3+1 general relativity and the gravitational wave signature of core-collapse supernovae PhD Thesis Universität Potsdam, Potsdam, Germany, http://nbn-resolving.de/urn/resolver.pl?urn=urn:nbn:de:kobv:517-opus-12986

[92] Bludman S A and van Riper K A 1977 Astrophys. J. 212 859-72

[93] Martí J M and Müller E 2003 Liv. Rev. Rel. 67

[94] Dimmelmeier H, Font J A and Müller E 2002 Astron. Astrophys. 388917

[95] Sedov L I 1959 Similarity and Dimensional Methods in Mechanics (New York: Academic Press)

[96] Tasker E J, Brunino R, Mitchell N L, Michielsen D, Hopton S, Pearce F R, Bryan G L and Theuns T 2008 Mon. Not. R. Astron. Soc. 390 1267-81

[97] Anninos P, Fragile P C and Salmonson J D 2005 Astrophys. J. 635723

[98] Oppenheimer J R and Volkoff G M 1939 Phys. Rev. 55374

[99] Petrich L I, Shapiro S L and Teukolsky S A 1986 Phys. Rev. D. 33 2100-10

[100] Gourgoulhon E 1993 Ann. Phys. 181

[101] Ott C D, Burrows A, Livne E and Walder R 2004 Astrophys. J. 600834

[102] Baumgarte T W, Shapiro S L and Shibata M 2000 Astrophys. J. Lett. 528 L29

[103] Fischer T, Whitehouse S C, Mezzacappa A, Thielemann F-K and Liebendörfer M 2009 Astron. Astrophys. 499 1-15

[104] Sumiyoshi K, Yamada S and Suzuki H 2007 Astrophys. J. 667382

[105] Liebendörfer M, Fischer T, Fröhlich C, Thielemann F-K and Whitehouse S 2008 J. Phys. G: Nucl. Part. Phys. 35014056

[106] Misner C W, Thorne K S and Wheeler J A 1973 Gravitation (San Francisco: Freeman) 